\title{
Interplay of the LHC and non-LHC dark matter searches in the effective field theory approach
}

\author{
Alexander Belyaev, ${ }^{1,2, *}$ Enrico Bertuzzo, ${ }^{3, \dagger}$ Cristian Caniu Barros, ${ }^{3, \$}$ Oscar Eboli, ${ }^{3, \S}$ \\ Giovanni Grilli di Cortona, ${ }^{3, \|}$ Fabio Iocco, ${ }^{4, \|}$ and Alexander Pukhov ${ }^{5, * *}$ \\ ${ }^{1}$ School of Physics and Astronomy, University of Southampton, Southampton SO17 1BJ, United Kingdom \\ ${ }^{2}$ Particle Physics Department, Rutherford Appleton Laboratory, \\ Didcot, Oxon OX11 0QX, United Kingdom \\ ${ }^{3}$ Instituto de Física, Universidade de São Paulo, C.P. 66.318, 05315-970 São Paulo, Brazil \\ ${ }^{4}$ ICTP South American Institute for Fundamental Research, and Instituto de Física Teórica-Universidade \\ Estadual Paulista (UNESP), Rua Dr. Bento Teobaldo Ferraz, 271, 01140-070 São Paulo, SP Brazil \\ ${ }^{5}$ Skobeltsyn Institute of Nuclear Physics, Moscow State University, Moscow 119992, Russia
}

(Received 22 July 2018; published 3 January 2019)

\begin{abstract}
We present accurate and up-to-date constraints on the complete set of dimension five and six operators with scalar, fermion and vector dark matter (DM). We find limits using LHC monojet data, spin independent and spin dependent direct searches, relic density and CMB, and show the interplay between high and low energy data in setting bounds on the parameter space. In order to properly compare data taken at different energies, we take into account the effect of the running and mixing of operators. We also take into account the local density uncertainties affecting direct detection data, and apply EFT validity criteria related to the cut on the invariant mass of DM pair production at the LHC, which turns out to be especially important for the case of vector DM. Finally, we estimate the potential of the future LHC runs to probe DM parameter space.
\end{abstract}

DOI: 10.1103/PhysRevD.99.015006

\section{INTRODUCTION}

Understanding the nature of dark matter (DM) is one of the greatest puzzles of modern particle physics and cosmology. Although overwhelming observational evidences from galactic to cosmological scales point to the existence of DM [1-3], after decades of experimental effort only its gravitational interaction has been experimentally confirmed. Currently, no information is available on the DM properties, such as its spin, mass, interactions other than gravitational, symmetry responsible for its stability, number of states associated to it, and possible particles that would mediate the interactions between DM and the standard model (SM) particles. If DM is light enough and interacts

\footnotetext{
*a.belyaev@soton.ac.uk

bertuzzo@if.usp.br

¥caniu@if.usp.br

§eboli@if.usp.br

"ggrilli@if.usp.br

iocco@ift.unesp.br

pukhov@lapp.in2p3.fr
}

Published by the American Physical Society under the terms of the Creative Commons Attribution 4.0 International license. Further distribution of this work must maintain attribution to the author(s) and the published article's title, journal citation, and DOI. Funded by SCOAP ${ }^{3}$. with SM particles directly or via some mediators with a strength beyond the gravitational one, its elusive nature can be detected or constrained in different ways:

(i) from direct production at colliders, resulting in a signature exhibiting an observed SM object, such as jet, Higgs, $Z$, or photon, that recoils against the missing energy from the DM pair [4-7];

(ii) via the relic density constraint obtained through the observations of cosmic microwave background (CMB) anisotropies, such as those of WMAP and PLANCK collaborations [1,8];

(iii) from DM direct detection (DD) experiments, which are sensitive to elastic spin independent (SI) or spin dependent (SD) DM scattering off nuclei [9-12];

(iv) from DM indirect detection searches, that look for SM particles produced in the decay or annihilation of DM present in the cosmos, both with high energies observables (gamma-rays, neutrinos, charge cosmic rays) produced in the local Universe [13-18], and by studying the effects of energy produced by DM annihilation in the early universe on the properties of the CMB spectrum $[1,19,20]$.

In this work we obtain the present constraints on three scenarios for the DM particles: complex scalars $(\phi)$, Dirac fermions $(\chi)$, and complex vectors $\left(V_{\mu}\right)$. In order to describe the interactions of the new states, we parametrize the DM 
TABLE I. Minimal basis of operators of dimension six or less involving only complex scalar DM $(\phi)$, Dirac fermion DM $(\chi)$ or complex vector DM $\left(V^{\mu}\right)$ interacting with SM quarks $(q)$ or gluons. Here we denote the field strength tensor of the gluons as $G^{\mu \nu}$ and its dual as $\tilde{G}^{\mu \nu}$. We show the Wilson coefficients at the scale $\Lambda$. Details on their running can be found in Sec. III.

\begin{tabular}{ll}
\hline \hline & Complex scalar DM \\
\hline$\frac{g_{*}^{2}}{\Lambda} \phi^{\dagger} \phi \bar{q} q$ & {$[\mathrm{C} 1]$} \\
$\frac{g_{*}^{2}}{\Lambda} \phi^{\dagger} \phi \bar{q} i \gamma^{5} q$ & {$[\mathrm{C} 2]$} \\
$\frac{g_{*}^{2}}{\Lambda^{2}} \phi^{\dagger} i \stackrel{\leftrightarrow}{\partial_{\mu}} \phi \bar{q} \gamma^{\mu} q$ & {$[\mathrm{C} 3]$} \\
$\frac{g_{*}^{2}}{\Lambda^{2}} \phi^{\dagger} i \stackrel{\leftrightarrow}{\partial_{\mu}} \phi \bar{q} \gamma^{\mu} \gamma^{5} q$ & {$[\mathrm{C} 4]$} \\
$\frac{g_{*}^{2}}{\Lambda^{2}} \phi^{\dagger} \phi G^{\mu \nu} G_{\mu \nu}$ & {$[\mathrm{C} 5]$} \\
$\frac{g_{*}^{2}}{\Lambda^{2}} \phi^{\dagger} \phi \tilde{G}^{\mu \nu} G_{\mu \nu}$ & {$[\mathrm{C} 6]$} \\
\hline
\end{tabular}

\begin{tabular}{lc}
\hline \hline & Dirac fermion DM \\
\hline$\frac{g_{*}^{2}}{\Lambda^{2}} \bar{\chi} \chi \bar{q} q$ & {$[\mathrm{D} 1]$} \\
$\frac{g_{*}^{2}}{\Lambda^{2}} \bar{\chi} i \gamma^{5} \chi \bar{q} q$ & {$[\mathrm{D} 2]$} \\
$\frac{g_{*}^{2}}{\Lambda^{2}} \bar{\chi} \chi \bar{q} i \gamma^{5} q$ & {$[\mathrm{D} 3]$} \\
$\frac{g_{*}^{2}}{\Lambda^{2}} \bar{\chi} \gamma^{5} \chi \bar{q} \gamma^{5} q$ & {$[\mathrm{D} 4]$} \\
$\frac{g_{*}^{2}}{\Lambda^{2}} \bar{\chi} \gamma^{\mu} \chi \bar{q} \gamma_{\mu} q$ & {$[\mathrm{D} 5]$} \\
$\frac{g_{*}^{2}}{\Lambda^{2}} \bar{\chi} \gamma^{\mu} \gamma^{5} \chi \bar{q} \gamma_{\mu} q$ & {$[\mathrm{D} 6]$} \\
$\frac{g_{*}^{2}}{\Lambda^{2}} \bar{\chi} \gamma^{\mu} \chi \bar{q} \gamma_{\mu} \gamma^{5} q$ & {$[\mathrm{D} 7]$} \\
$\frac{g_{*}^{2}}{\Lambda^{2}} \bar{\chi} \gamma^{\mu} \gamma^{5} \chi \bar{q} \gamma_{\mu} \gamma^{5} q$ & {$[\mathrm{D} 8]$} \\
$\frac{g_{*}^{2}}{\Lambda^{2}} \bar{\chi} \sigma^{\mu \nu} \chi \bar{q} \sigma_{\mu \nu} q$ & [D9] \\
$\frac{g_{*}^{2}}{\Lambda^{2}} \bar{\chi} \sigma^{\mu \nu} i \gamma^{5} \chi \bar{q} \sigma_{\mu \nu} q$ & [D10] \\
\hline \hline
\end{tabular}

Complex vector DM

\begin{tabular}{lc}
$\frac{g_{*}^{2} m_{\mathrm{MM}}^{2}}{\Lambda^{3}} V_{\mu}^{\dagger} V^{\mu} \bar{q} q$ & {$[\mathrm{~V} 1]$} \\
$\frac{g_{*}^{2} m_{\mathrm{DM}}^{2}}{\Lambda^{3}} V_{\mu}^{\dagger} V^{\mu} \bar{q} i \gamma^{5} q$ & {$[\mathrm{~V} 2]$} \\
$\frac{g_{*}^{2} m_{\mathrm{DM}}^{2}}{2 \Lambda^{4}} i\left(V_{\nu}^{\dagger} \partial_{\mu} V^{\nu}-V^{\nu} \partial_{\mu} V_{\nu}^{\dagger}\right) \bar{q} \gamma^{\mu} q$ & {$[\mathrm{~V} 3]$} \\
$\frac{g_{*}^{2} m_{\mathrm{DM}}^{2}}{2 \Lambda^{4}}\left(V_{\nu}^{\dagger} \partial_{\mu} V^{\nu}-V^{\nu} \partial_{\mu} V_{\nu}^{\dagger}\right) \bar{q} i \gamma^{\mu} \gamma^{5} q$ & {$[\mathrm{~V} 4]$} \\
$\frac{g_{*}^{2} m_{\mathrm{DM}}^{2}}{\Lambda^{3}} V_{\mu}^{\dagger} V_{\nu} \bar{q} i \sigma^{\mu \nu} q$ & {$[\mathrm{~V} 5]$} \\
$\frac{g_{*}^{2} m_{\mathrm{M}}^{2}}{\Lambda^{3}} V_{\mu}^{\dagger} V_{\nu} \bar{q} \sigma^{\mu \nu} \gamma^{5} q$ & {$[\mathrm{~V} 6]$} \\
$\frac{g_{*}^{2} m_{\mathrm{DM}}}{2 \Lambda^{3}}\left(V_{\nu}^{\dagger} \partial^{\nu} V_{\mu}+V_{\nu} \partial^{\nu} V_{\mu}^{\dagger}\right) \bar{q} \gamma^{\mu} q$ & {$[\mathrm{V7P}]$} \\
$\frac{g_{*}^{2} m_{\mathrm{DM}}^{2}}{2 \Lambda^{4}}\left(V_{\nu}^{\dagger} \partial^{\nu} V_{\mu}-V_{\nu} \partial^{\nu} V_{\mu}^{\dagger}\right) \bar{q} i \gamma^{\mu} q$ & {$[\mathrm{~V} 7 \mathrm{M}]$} \\
$\frac{g_{*}^{2} m_{\mathrm{DM}}}{2 \Lambda^{3}}\left(V_{\nu}^{\dagger} \partial^{\nu} V_{\mu}+V_{\nu} \partial^{\nu} V_{\mu}^{\dagger}\right) \bar{q} \gamma^{\mu} \gamma^{5} q$ & {$[\mathrm{~V} 8 \mathrm{P}]$} \\
$\frac{g_{*}^{2} m_{\mathrm{MM}}^{2}}{2 \Lambda^{4}}\left(V_{\nu}^{\dagger} \partial^{\nu} V_{\mu}-V_{\nu} \partial^{\nu} V_{\mu}^{\dagger}\right) \bar{q} i \gamma^{\mu} \gamma^{5} q$ & {$[\mathrm{~V} 8 \mathrm{M}]$} \\
$\frac{g_{*}^{2} m_{\mathrm{DM}}}{2 \Lambda^{3}} \epsilon^{\mu \nu \rho \sigma}\left(V_{\nu}^{\dagger} \partial_{\rho} V_{\sigma}+V_{\nu} \partial_{\rho} V_{\sigma}^{\dagger}\right) \bar{q} \gamma_{\mu} q$ & {$[\mathrm{V9P}]$} \\
$\frac{g_{*}^{2} m_{\mathrm{DM}}}{2 \Lambda^{3}} \epsilon^{\mu \nu \rho \sigma}\left(V_{\nu}^{\dagger} \partial_{\rho} V_{\sigma}-V_{\nu} \partial_{\rho} V_{\sigma}^{\dagger}\right) \bar{q} i \gamma_{\mu} q$ & {$[\mathrm{~V} 9 \mathrm{M}]$} \\
$\frac{g_{*}^{2} m_{\mathrm{DM}}}{2 \Lambda^{3}} \epsilon^{\mu \nu \rho \sigma}\left(V_{\nu}^{\dagger} \partial_{\rho} V_{\sigma}+V_{\nu} \partial_{\rho} V_{\sigma}^{\dagger}\right) \bar{q} \gamma_{\mu} \gamma^{5} q$ & {$[\mathrm{~V} 10 \mathrm{P}]$} \\
$\frac{g_{*}^{2} m_{\mathrm{DM}}}{2 \Lambda^{3}} \epsilon^{\mu \nu \rho \sigma}\left(V_{\nu}^{\dagger} \partial_{\rho} V_{\sigma}-V_{\nu} \partial_{\rho} V_{\sigma}^{\dagger}\right) \bar{q} i \gamma_{\mu} \gamma^{5} q$ & {$[\mathrm{~V} 10 \mathrm{M}]$} \\
$\frac{g_{*}^{2} m_{\mathrm{DM}}^{2}}{\Lambda^{4}} V_{\mu}^{\dagger} V^{\mu} G^{\rho \sigma} G_{\rho \sigma}$ & {$[\mathrm{V} 11]$} \\
$\frac{g_{*}^{2} m_{\mathrm{DM}}^{2}}{\Lambda^{4}} V_{\mu}^{\dagger} V^{\mu} \tilde{G}^{\rho \sigma} G_{\rho \sigma}$ & {$[\mathrm{V} 12]$} \\
\hline \hline
\end{tabular}

interactions with the SM quarks and gluons as an effective field theory (EFT) that contains a complete set of operators of dimension six or less. Notice the presence of the coupling $g_{*}$ in the definition of the effective operators, which we insert according to the naive dimensional analysis [21]. Moreover, for the vector DM case we choose the parametrization suggested in Ref. [22] that takes into account the high energy behaviour of the scattering amplitudes that are enhanced by an energy factor $\left(E / m_{\mathrm{DM}}\right)$ for every longitudinal vector DM polarization.

Here, our goal is to explore the complementarity of the collider and noncollider experiments mentioned above for all DM EFT operators and DM spin listed in Table I [22-24] for DM masses in the GeV-TeV range; for lighter DM masses see e.g., [25]. For the sake of consistency of our analyses, we obtain the present constraints from the LHC data taking into account the validity of the EFT [26-28] using the prescription of Ref. [29]; see Sec. IV for details. Furthermore, the correct comparison between the LHC and noncollider bounds requires that we consider in our analyses the running of the EFT operators from the $\mathrm{TeV}$ scale down to the $\mathrm{GeV}$ one. This is important because the running of operators leads to mixing between them at low energy which can give rise to stronger DM DD limits [30-36].

As it is well known, the DM DD searches are plagued by the uncertainty on the local DM density [37-42] which propagates to the limits on the DM DD cross sections reported by the experiments, inducing variations up to one order of magnitude. Here we estimate the impact of this uncertainty on the DM bounds, presenting three scenarios that range from a conservative to a more optimistic one. On the other hand, indirect constraints from CMB are unaffected by the usual unknowns related to the DM density profile within structures. For this reason, we also include the $\mathrm{CMB}$ data in our analyses that lead to more robust limits.

This paper is organized as follows: in Sec. III we study the running of the EFT DM operators from the $\mathrm{TeV}$ to the $\mathrm{GeV}$ scale and the effect of their mixing, while we present our analyses framework and available constraints in Sec. IV. Section V contains our main results for all operators in Table I that show the complementarity of the collider and non-collider constraints. Finally, we draw our conclusions in Sec. VI.

\section{THEORETICAL SETUP}

In this work, we assume that for all the operators from Table I the DM current is coupled with the same strength to the SM currents for all quark flavors or the gluon field strength (and its dual). This assumption is specific for scalar and pseudoscalar SM currents since an alternative approach can also take place, where the respective couplings contain quark masses as an additional factor in the 
form of $m_{q} / \Lambda$ (see, e.g., [23,43]). In our study we take into account (and it is important to do this) that operators with scalar or pseudoscalar quark currents (such as C1, C2, D1, D2, D3, D4, V1, and V2) run with the QCD scale as quark mass, such that at low energies the operators scale as

$$
\frac{1}{\Lambda^{n}} \rightarrow \frac{m_{q}(\mu)}{m_{q}\left(\mu_{L H C}\right)} \frac{1}{\Lambda^{n}}
$$

where $\mu_{L H C} \sim \mathrm{TeV}$ is the scale at which the operator is generated and $n=1,2$ is a coefficient to get the correct dimension for the different operators. One should stress that the ratio of Wilson coefficients is scale-independent since all quark masses are renormalized by the same factor. We also take into account the analogous running of the gluon operators

$$
\frac{1}{\Lambda^{n}} \rightarrow \frac{\alpha_{s}(\mu)}{\alpha_{s}\left(\mu_{L H C}\right)} \frac{1}{\Lambda^{n}}
$$

in spite of the fact that they do not contain explicit $\alpha_{s}$ dependence.

Let us note that the operators for vector DM have also additional factor $\left(\frac{m_{\mathrm{DM}}}{\Lambda}\right)^{n}(n=1,2$-depending on the operator) which provides the same high-energy asymptotic for all operators with the fixed power of $\Lambda$ as discussed in Ref. [22].

In our framework we do not consider operators where the DM couples to leptons and electroweak bosons without loss of generality. Such operators are not expected to introduce new leading signatures at the LHC, but could significantly lower down relic density and respectively increase the lower limit on $\Lambda$. On the other hand relic density can be strongly decreased by additional BSM content which could be involved into coannihilation with DM or by nonthermal (e.g., freeze-in) origin of DM which will also lead to increase of the upper limit on $\Lambda$. Therefore various model assumptions could lead to the same increase of the lower limit on $\Lambda$ and therefore larger values of $\Lambda$ should not be excluded in general by relic density constraints. From this point of view we find the conservative (minimal value) of the upper limit on $\Lambda$ from the relic density evaluation and compare it with the lower limit on $\Lambda$ from DM direct detection and LHC data.

\section{DIRECT DETECTION AND RUNNING EFFECT OF THE EFT OPERATORS}

In this section we demonstrate the importance of the running of the operators for the DM DD constraints. As we know, in quantum field theory radiative corrections may be important to properly assess the phenomenological implications of a model. In the case of DM EFT this is even more so, because (i) the bounds on the parameter space involve experiments with very different energy scales, and (ii) the Wilson coefficients can vary substantially between the typical LHC energies (a few TeV) and the typical energies
TABLE II. Operators for WIMP-nucleon SI interactions. Even and odd refer to the properties with respect to quark/antiquark exchange.

\begin{tabular}{cccc}
\hline \hline & WIMP spin & Even operators & Odd operators \\
\hline SI & 0 & $4 m_{\mathrm{DM}} \phi \phi^{\dagger} \bar{N} N$ & $2 i\left(\partial_{\mu} \phi \phi^{\dagger}-\phi \partial_{\mu} \phi^{\dagger}\right) \bar{N} \gamma^{\mu} N$ \\
& $1 / 2$ & $2 \bar{\chi} \chi \bar{N} N$ & $2 \bar{\chi} \gamma_{\mu} \chi \bar{N} \gamma^{\mu} N$ \\
& 1 & $4 m_{\mathrm{DM}} V_{\mu}^{*} V^{\mu} \bar{N} N$ & $2 i\left(V^{\dagger \alpha} \partial_{\mu} V_{\alpha}-V^{\alpha} \partial_{\mu} V_{\alpha}^{\dagger}\right) \bar{N} \gamma^{\mu} N$ \\
\hline \hline
\end{tabular}

of DM DD experiments (below the $\mathrm{GeV}$ ). The first radiative effects to be considered were the QCD ones [30-32], while only later it has been realized that EW loops could be important as well [33-36,44].

In our discussion we will make use of the renormalization of currents [32], which we found fits best the purpose of our study. As can be seen from Table I, all the operators are written as a product

$$
\mathcal{O}=J_{\chi}^{A} J_{\mathrm{SM}}^{A},
$$

where $A$ denotes some combination of Lorentz indexes and $J_{\chi}$ and $J_{\mathrm{SM}}$ are currents constructed out of DM and SM fields only. Since we suppose $\chi$ (and hence $J_{\chi}$ ) to be a gauge singlet, only the renormalization of the SM currents has to be computed. The lowest dimensional currents considered in this work are the dimension 3 and 4 operators ${ }^{1}$

$$
\begin{aligned}
& J_{d=3}=\left\{\bar{q} \gamma^{\mu} q, \bar{q} \gamma^{\mu} \gamma_{5} q, \bar{q} q, \bar{q} \gamma_{5} q\right\}, \\
& J_{d=4}=\left\{G_{\mu \nu} G^{\mu \nu}, \tilde{G}_{\mu \nu} G^{\mu \nu}\right\},
\end{aligned}
$$

where $q$ denotes any of the SM quarks and $G$ is the gluon field. At the low energies involved in the DM DD experiments, the relevant degrees of freedom are nucleons and nuclei, rather than quarks and gluons. For this reason, we should match the amplitudes involving quarks and gluons with matrix elements involving nucleons (see e.g., [45,46] and Table II for some of the operators).

Let us start with the scalar quark current. The matrix elements at zero momentum transfer are $[47,48]$

$$
\begin{aligned}
\left\langle N\left|m_{q}(\mu) \bar{q} q\right| N\right\rangle & =m_{N} f_{T q}^{(N)}, \\
\left\langle N\left|m_{Q}(\mu) \bar{Q} Q\right| N\right\rangle & =\frac{2}{27} m_{N} f_{T G}^{(N)},
\end{aligned}
$$

for light $(q=u, d, s)$ and heavy quarks $(Q=c, b, t)$, respectively. In the above equation, $N$ stands either for proton $p$ or neutron $n$. The quantity $f_{T q}^{(N)}$ amounts to the light quark contribution to the nucleon mass $m_{N}$, while $f_{T G}^{(N)}=1-\sum_{q} f_{T q}^{(N)}$. Notice that both the quark condensate

\footnotetext{
${ }^{1}$ There are two dimension 2 currents which are gauge singlets, $B_{\mu \nu}$ and $H^{\dagger} H$, which however do not mix under renormalization.
} 
and the running mass depend on the scale $\mu$, in such a way that their product is scale independent. As a consequence, the form factors are also scale independent. The light quarks form factors are known from hadron spectroscopy and lattice calculations. In our numerical analysis we use the quark scalar form factors presented in micrOMEGAs [49]. Using the matrix elements of Eq. (5) the DM-nucleon spin independent (SI) cross section can be written as

$$
\sigma^{S I}=\frac{4 \lambda^{2}}{\pi}\left(\frac{m_{N} m_{\mathrm{DM}}}{m_{N}+m_{\mathrm{DM}}}\right)^{2},
$$

where $\lambda$ is an operator dependent coefficient that will be defined below.

To discuss the importance of the running, let us discuss the example of the $\mathrm{C} 1$ operator. We will suppose that such operator is generated at the scale $\Lambda \sim \mu_{L H C} \sim \mathrm{TeV}$ with Wilson coefficient $g_{*}^{2}$. As already explained, the $\bar{q} q$ operator runs as the inverse of the quark mass, in such a way that the $\mathrm{C} 1$ operator at an arbitrary scale $\mu$ looks like

$$
\frac{g_{*}^{2}}{\Lambda}\left(\phi^{\dagger} \phi\right)\left(\sum_{q=u, d, s, c, b, t} \frac{m_{q}(\mu)}{m_{q}\left(\mu_{L H C}\right)}(\bar{q} q)\right) .
$$

The same is true for the D1 and V1 operators, and the $\lambda$ coefficient of Eq. (6) reads

$$
\begin{aligned}
& \lambda_{C 1}^{(N)}=\frac{m_{N} g_{*}^{2}}{4 m_{\mathrm{DM}} \Lambda}\left[\sum_{q} \frac{f_{T q}^{(N)}}{m_{q}\left(\mu_{L H C}\right)}+\frac{2}{27} f_{T G}^{(N)} \sum_{Q} \frac{1}{m_{Q}\left(\mu_{L H C}\right)}\right], \\
& \lambda_{D 1}^{(N)}=\frac{m_{N} g_{*}^{2}}{2 \Lambda^{2}}\left[\sum_{q} \frac{f_{T q}^{(N)}}{m_{q}\left(\mu_{L H C}\right)}+\frac{2}{27} f_{T G}^{(N)} \sum_{Q} \frac{1}{m_{Q}\left(\mu_{L H C}\right)}\right], \\
& \lambda_{V 1}^{(N)}=\frac{m_{N} m_{\mathrm{DM}}^{2} g_{*}^{2}}{4 \Lambda^{3}}\left[\sum_{q} \frac{f_{T q}^{(N)}}{m_{q}\left(\mu_{L H C}\right)}+\frac{2}{27} f_{T G}^{(N)} \sum_{Q} \frac{1}{m_{Q}\left(\mu_{L H C}\right)}\right],
\end{aligned}
$$

where $N=n, p$.

A similar effect is present for the operators involving the $G^{\mu \nu} G_{\mu \nu}$ current (C5 and V11). In this case, the matrix element of the gluon current is $[47,48]$

$$
\left\langle N\left|\alpha_{s}(\mu) G_{\mu \nu} G^{\mu \nu}\right| N\right\rangle=-\frac{8 \pi}{9} m_{N} f_{T G}^{(N)},
$$

and the combination $\alpha_{s}(\mu) G_{\mu \nu} G^{\mu \nu}$ (and hence the form factor) is scale independent. Suppose now that the C5 operator is generated at the $\Lambda$ scale with Wilson coefficient $g_{*}^{2}$. At an arbitrary scale $\mu$, the operator looks like

$$
\frac{\alpha_{s}(\mu)}{\alpha_{s}\left(\mu_{L H C}\right)} \frac{g_{*}^{2}}{\Lambda^{2}}\left(\phi^{\dagger} \phi\right)\left(G_{\mu \nu} G^{\mu \nu}\right)
$$

The same result applies to the V11 operator. The $\lambda$ coefficients of Eq. (6) reads

$$
\begin{aligned}
& \lambda_{C 5}^{(N)}=-\frac{2 \pi}{9} g_{*} f_{T G}^{(N)} \frac{m_{N}}{\alpha_{s}\left(\mu_{L H C}\right) \Lambda^{2} m_{\mathrm{DM}}}, \\
& \lambda_{V 11}^{(N)}=-\frac{2 \pi}{9} g_{*} f_{T G}^{(N)} \frac{m_{N} m_{\mathrm{DM}}}{\alpha_{s}\left(\mu_{L H C}\right) \Lambda^{4}} .
\end{aligned}
$$

In principle, in addition to the running of the individual Wilson coefficients, a mixing between operators is generated [30-33]. However, for operators involving the $\left\{m_{q} \bar{q} q, G_{\mu \nu} G^{\mu \nu}\right\}$ currents the mixing was found to be numerically unimportant [33].

Let us now turn to the analysis of the $\left\{\bar{q} \gamma^{\mu} q, \bar{q} \gamma^{\mu} \gamma_{5} q\right\}$ currents. In this case, the mixing between operators can be numerically important. Let us consider for instance the operators involving the $\bar{q} \gamma^{\mu} \gamma_{5} q$ axial vector current, i.e., the operators C4, D7-D8, V4. These operators are responsible for spin dependent DM scattering at DD experiments, with bounds much weaker than those of spin independent experiments. Nonetheless, the $\bar{q} \gamma^{\mu} \gamma_{5} q$ axial vector current mixes with the $\bar{q} \gamma^{\mu} q$ vector current during the running, and a SI cross section is generated [33-35]. Suppose for instance the $\mathrm{C} 4$ operator is generated at the $\Lambda$ scale above the top mass

$$
\mathcal{L}=\frac{g_{*}^{2}}{\Lambda^{2}}\left(\phi^{\dagger} i \stackrel{\leftrightarrow}{\partial_{\mu}} \phi\right)\left[\sum_{q}\left(\bar{q} \gamma^{\mu} \gamma^{5} q\right)+\sum_{Q}\left(\bar{Q} \gamma^{\mu} \gamma^{5} Q\right)\right],
$$

where the sum is taken over all light $(q)$ and heavy $(Q)$ quark flavors. Then, using the results of Refs. [33-35], the operators present in the Lagrangian at the DD scale are

$$
\begin{aligned}
\mathcal{L} \simeq & {\left[1-\frac{3 \alpha_{t}}{2 \pi} \log \left(\frac{\mu_{L H C}}{m_{t}}\right)+\cdots\right] \frac{g_{*}^{2}}{\Lambda^{2}}\left(\phi^{\dagger} i \stackrel{\leftrightarrow}{\partial_{\mu}} \phi\right)\left(\bar{u} \gamma^{\mu} \gamma_{5} u\right) } \\
& +\left[1+\frac{3 \alpha_{t}}{2 \pi} \log \left(\frac{\mu_{L H C}}{m_{t}}\right)+\cdots\right] \frac{g_{*}^{2}}{\Lambda^{2}}\left(\phi^{\dagger} i \stackrel{\leftrightarrow}{\partial_{\mu}} \phi\right)\left(\bar{d} \gamma^{\mu} \gamma_{5} d+\bar{s} \gamma^{\mu} \gamma_{5} s\right) \\
& +\left(3-8 s_{w}^{2}\right)\left[\frac{\alpha_{t}}{2 \pi} \log \left(\frac{\mu_{L H C}}{m_{t}}\right)+\cdots\right] \frac{g_{*}^{2}}{\Lambda^{2}}\left(\phi^{\dagger} i \stackrel{\leftrightarrow}{\partial_{\mu}} \phi\right)\left(\bar{u} \gamma^{\mu} u\right) \\
& +\left(3-4 s_{w}^{2}\right)\left[-\frac{\alpha_{t}}{2 \pi} \log \left(\frac{\mu_{L H C}}{m_{t}}\right)+\cdots\right] \frac{g_{*}^{2}}{\Lambda^{2}}\left(\phi^{\dagger} i \stackrel{\leftrightarrow}{\partial_{\mu}} \phi\right)\left(\bar{d} \gamma^{\mu} d+\bar{s} \gamma^{\mu} s\right),
\end{aligned}
$$


where we show only the most relevant contribution, coming from top loops. In the previous expression, $\alpha_{t} \equiv y_{t}^{2} / 4 \pi$, with $y_{t}$ the top Yukawa coupling. Notice that the top contribution is present only down to the top scale, where the top quark is integrated out. As already stressed, in addition to the SD operators of the first two lines, the running has generated the SI operators of the last two lines. Even though the Wilson coefficients of the SI operators are smaller than those of the SD ones, it has been shown in Ref. [35] that they are sufficient to put bounds on $g_{*}^{2} / \Lambda^{2}$ which are up to a factor of 100 stronger with respect to the typical bounds that can be obtained from SD experiments (i.e., considering only the operators in the first two lines). This shows clearly the importance of the running in setting consistent bounds on the parameter space of the DM EFT. Let us point out however that for the effect to be numerically relevant, it is instrumental for the coupling between the DM and the top currents to be switched on in Eq. (13). If this is not the case, the SI operators are still generated in the running, but with much smaller Wilson coefficients and weaker bounds (see Ref. [35] for more details). In the analysis of Sec. V we will numerically implement the running and the mixing of the currents using the runDM code [33-35,50], which takes into account all the contributions.

We also perform analysis of DM direct detection signals from operators whose contribution is momentum suppressed. These operators vanish in the limit of zero momentum transfer limit, but they are not exactly negligible at actual DM DD experimental transferred energies in $10-100 \mathrm{KeV}$ energy range. We have used XENON100 constraints [51] on the relevant non-relativistic nucleon operators $\mathcal{O}_{6}, \mathcal{O}_{7}, \mathcal{O}_{10}, \mathcal{O}_{11}, \mathcal{O}_{12}$ and Refs. [46,52] to translate them into limits on C4, C6, D2, and D10 operators for scalar and fermions DM. Since XENON100 are very sensitive only to $\mathcal{O}_{10}, \mathcal{O}_{11}$, and $\mathcal{O}_{12}$ nucleon operators, the only non-negligible constraints can be set on D2 and D10 operators as we discuss below in Sec. V. Since, to the best of our knowledge, there is no studies on connection of vector DM operators to nonrelativistic operators, we did not produce the respective limits of vector DM.

\section{ANALYSIS SETUP AND CONSTRAINTS}

In this section we describe the analysis setup and constraints used in this study. In particular we delineate the limits originating from $\mathrm{CMB}$, direct detection experiments, and collider searches.

Direct and indirect detection constraints are affected by uncertainties of astrophysical nature. On one hand, the scattering of DM off nuclei on the Earth depends on the DM local density and velocity distributions around Earth. On the other hand, the DM self-annihilation rate in our galaxy depends on its particle density distribution therein. For what is concern of this paper, whenever possible we make the conservative choice to select targets that can reduce as much as possible the uncertainties, and thoroughly account for the remaining ones. In practice, this means that: (i) for indirect searches we adopt CMB limits, as the energy injection of DM annihilation is unaffected by the usual unknowns related to DM density profile within structures; (ii) for direct searches we explicitly take into account the systematic effects generated by the astrophysical uncertainties in the determination of the local DM density $\rho_{0}$.

\section{A. CMB constraints}

The observation of byproducts of DM annihilation (or decay) in astrophysical targets can be used to determine (or constrain, in case of missing observations) relevant DM properties such as its mass and annihilation cross section. Such bounds depend on the unknown DM distribution within the astrophysical objects chosen as targets. A detailed analysis has shown that, choosing the CMB as target, the leading signal of DM annihilation is produced around redshift $z \sim 600$ [53]. This makes the CMB a quantitatively competitive channel for indirect searches $[1,19,20]$, since at $z \sim 600$ the DM has not fallen into structures yet, and the observation is free from the usual astrophysical sources of uncertainties (density profile within a halo, distribution and density of subhalos, mass of the smallest bound halo). Moreover, additional sources of systematics affecting the CMB constraints have also been thoroughly examined [54,55], and shown to affect the results below the sensitivity needed for this paper. In the following, we will neglect them leaving our conclusions unaltered.

In order to set the CMB bounds on the quantity of our interest $\Lambda$, we first obtain the observational bound on the thermally averaged annihilation cross-section at redshift 600:

$$
p_{\mathrm{ann}}=\sum_{j} f_{j}\left(600, m_{\mathrm{DM}}\right) \frac{\langle\sigma v\rangle_{j}(600)}{m_{\mathrm{DM}}},
$$

where $\langle\sigma v\rangle_{j}(600)$ is the thermally averaged partial annihilation cross section for the $j$ th channel at redshift 600 and $f_{j}\left(z, m_{\mathrm{DM}}\right)$ is the fraction of annihilation energy that is absorbed by the plasma at redshift $z$. The quantity $p_{\text {ann }}$ is constrained by Planck TT, TE, EE, and lowP data [1]:

$$
p_{\text {ann }}<4.1 \times 10^{-28} \frac{\mathrm{cm}^{3}}{\mathrm{~s} \mathrm{GeV}} \quad \text { at } 95 \% \text { C.L. }
$$

and the values of the variable $f_{j}\left(z, m_{\mathrm{DM}}\right)$ are taken form Ref. [56].

In order to bound the new physics scale $\Lambda$, we numerically compute the velocity dependent annihilation cross section with micrOMEGAs [45], obtaining the relationship between $\langle\sigma v\rangle_{j}(600)$ and $\Lambda$ for each effective operator and each final state. It may be noted that for those operators for 
which the s-wave process is dominating, the thermally averaged cross section is constant ${ }^{2}$ and $\langle\sigma v\rangle_{j}(600)=$ $\langle\sigma v\rangle_{j}(0)$. On the other hand, for those operators in which the $\mathrm{p}$-wave contribution dominates the annihilation cross section, the CMB bound is almost ineffective, since it is suppressed by the low $T_{\mathrm{CMB}}$ temperature. A possible bound in this case can be obtained from the big bang nucleosynthesis (BBN), since this process happens at $T_{B B N} \sim \mathrm{MeV}$. As shown in Refs. [57,58], the bounds on $\langle\sigma v\rangle$ are generically weaker than those obtained from the CMB, and we have explicitly checked that in all the cases in which the CMB bound is ineffective, the BBN bound is also not relevant.

\section{B. DM direct detection constraints}

The determination of the DM mass and elastic scattering cross section in a DM DD experiments is affected by uncertainties associated to the flux of DM particles crossing the Earth. Although the uncertainties on the Sun's relative motion with respect to the Galactic Center, or the exact morphology of the Galactic bulge, do not affect the conclusion of the presence of a sizable component of DM at the Sun's location [37], they impact the reconstruction of the DM profile throughout the Milky Way [38]. More specifically, two sources of uncertainties are relevant for DM DD experiments: the local DM density ${ }^{3}$ and velocity distribution [40-42,59]. Although relevant, their effect is often overlooked in putting DD bounds on the parameter space of a model. The shape of the velocity distribution is known to sizeably affect the results of different types of detectors through their sensitivity to different DM masses. In particular, it is important to note the large uncertainty in the tail of the velocity distribution which is essential for DD uncertainty especially for low DM masses. Based on results from cosmological simulations of galaxy formation, it has been recently argued that the shape of the velocity distribution is well approximated by a Maxwell-Boltzmann one. This is so especially when baryonic processes are taken into account (in the numerical simulations of Galaxy formation in cosmological context) and in spite of the remarkable variation in the central value of the distribution as a function of the halo mass, e.g., Ref. [42]. It has also been recently shown that the effect of variation of DM DD rates from the variation of the central value of such distribution can be overcome by the variation of the Galactic uncertainties, see Ref. [60]. For the purpose of

\footnotetext{
${ }^{2}$ We remind the reader that the thermally averaged cross section is given by

$$
\langle\sigma v\rangle=\left\langle a+b v^{2}+\cdots\right\rangle=a+\frac{3}{2} b \frac{T}{m_{\mathrm{DM}}}+\cdots
$$

with $T$ the temperature at which the process is computed.

${ }^{3}$ See Ref. [39] for a recent and thorough review.
}

this paper, we have decided to follow closely the analysis in Ref. [60]. In particular, we consider three possible values $\rho_{0}=0.06 \mathrm{GeV} / \mathrm{cm}^{3}, \rho_{0}=0.3 \mathrm{GeV} / \mathrm{cm}^{3}$ and $\rho_{0}=$ $1.8 \mathrm{GeV} / \mathrm{cm}^{3}$. These values correspond to the lowest and highest possible values of local relic density $\rho_{0}$, resulting from the uncertainties in the determination of $R_{0}$ and $v_{0}$, the values of Galactic parameters, [60], while the central value of $\rho_{0}$ is used by the experimental collaborations. Using this range for $\rho_{0}$ we apply the experimental bounds stemming from Xenon1T SI searches [61] and from PandaX-II SD analyses [62]. In our analysis we not take into account the effects of the possible existence of a dark disk or stellar streams with associated dark matter content, which are expected to affect the low and high end of the velocity distribution respectively, but whose actual existence is currently debated, and virtually impossible to account for correctly as of today.

We have also taken into account uncertainties in XENON1T DM DD limit related to the different detector sensitivity for different $v_{0}$ values as well as values of the escape velocity, $v_{\text {esc }}$ and found non-negligible effects for $m_{\mathrm{DM}}$ below $10 \mathrm{GeV}$ which lead to modification of the bounds on $\Lambda$ by a factor up to two. To find these uncertainties we follow the following procedure. The values of the circular velocity $v_{0}$ corresponding to the determination of $\rho_{0}=0.06 \mathrm{GeV} / \mathrm{cm}^{3}$, and $1.8 \mathrm{GeV} / \mathrm{cm}^{3}$ where $v_{0}=180 \mathrm{~km} / \mathrm{s}$, and $312 \mathrm{~km} / \mathrm{s}$, respectively, see [60]. For central value of $\rho_{0}=0.3 \mathrm{GeV} / \mathrm{cm}^{3}$ we use value $v_{0}=220 \mathrm{~km} / \mathrm{s}$ which is usually adopted by most Direct Detection collaborations. DM DD limit for low DM masses is especially sensitive to the value maximal velocity $v_{\text {esc }}$ of DM particles related to the finite value galactic mass. The value varies within the $(498 \mathrm{~km} / \mathrm{s}-608 \mathrm{~km} / \mathrm{s})$ range [40]. For our analysis we have chosen $v_{\text {esc }}=498 \mathrm{~km} / \mathrm{s}$ because it provides more conservative exclusion. Using results of Xenon1T experiment [61] obtained for $v_{0}=220 \mathrm{~km} / \mathrm{s}$ we have recalculated limits for $v_{0}=180 \mathrm{~km} / \mathrm{s}$ and $v_{0}=312 \mathrm{~km} / \mathrm{s}$ which we present in Fig. 1 . In case of

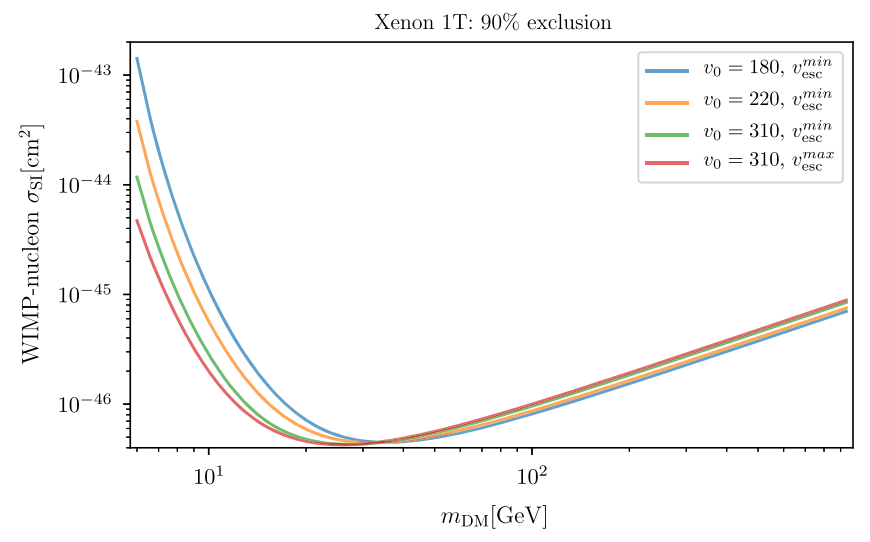

FIG. 1. Variation of DM DD limits from XENON 1T recalculated for different $v_{0}$ and $v_{\text {esc }}$ velocities for $\left(v_{\mathrm{esc}}^{\min }, v_{\mathrm{esc}}^{\max }\right)=$ $(498 \mathrm{~km} / \mathrm{s}, 608 \mathrm{~km} / \mathrm{s})$. 
$v_{0}=312 \mathrm{~km} / \mathrm{s}$ we also show the limit obtained for $v_{\text {esc }}=608 \mathrm{~km} / \mathrm{s}$. For smaller $v_{0}$ dependence on this parameter is not too strong. One can see that for small DM masses of the order of $5 \mathrm{GeV}$ the XENON1T limit on the cross section fluctuates by factor of 5 upwards for small values of $v_{0}$ (low values of $\rho_{0}$ ) and by factor 5 downwards for large values of $v_{0}$ (large values of $\rho_{0}$ ).

In addition, when we obtain DD bounds, we also take into account the relic density constraint. Direct detection experiments constrain DM particles assuming that their relic density matches the one of the cold DM component. The simplest way to compute the detection rates is to rescale the DM density distribution according to the prescription

$$
\rho_{i} \equiv \rho_{0} \min \left(1, \frac{\Omega_{i}}{\Omega_{\mathrm{DM}}}\right), \quad i=\phi, \chi, V,
$$

where $\rho_{0}$ is the local DM density, $\Omega_{\chi}$ is the theoretical relic density obtained via micrOMEGAs for every operator listed in Table I and $\Omega_{\mathrm{DM}} \simeq 0.12$ from Ref. [1].

\section{Collider constraints}

In the upcoming sections, we also present bounds coming from collider searches. In order to perform the required simulations, we implemented the different effective operators independently in FeynRules [63] and LANHeP [64] and generated the signal using MADGRAPH5_AMC@NLO [65]. The hadronization and parton showering was done using PYTHIA 6.4 [66], with subsequent detector simulation performed using MADANALYSIS5 [67] and DELPHES [68].

In order to obtain the limits on the scale $\Lambda$ we consider the CMS analysis of final state presenting jets and missing transverse energy [5] based on data obtained at $13 \mathrm{TeV}$ with an integrated luminosity of $35.9 \mathrm{fb}^{-1}$. This analysis was performed as a counting experiment in 22 independent signal regions characterized by (i) $E_{T}^{\text {miss }}>250 \mathrm{GeV}$, (ii) one jet with $p_{T}^{\text {jet }}>100 \mathrm{GeV}$ and (iii) $\left|\eta_{j}\right|<4$.5. In order to simulate the DM contribution to this process we studied

$$
p p \rightarrow j+(\phi \phi, \chi \chi, V V)
$$

for all operators in Table I. As it is well known, higher dimensional operators such as the ones in our EFT can lead to perturbative partial wave unitarity violation at high energies, signaling a maximum value of the center of mass energy for its applicability. Therefore, in order to guarantee the validity of the EFT we impose in our simulation that the invariant mass of the DM pair $M_{\chi \chi, \phi \phi, V V}$ satisfies [29]:

$$
M_{\chi \chi, \phi \phi, V V}<\Lambda .
$$

In our statistical analysis we use the simplified likelihood approach given in Ref. [69], taking into account the full correlation and covariance matrix provided in [5]. More specifically, we defined the likelihood function

$$
\begin{aligned}
\mathcal{L}\left(\Lambda, g_{*}, \theta\right)= & \prod_{i} \frac{\left(s_{i}\left(\Lambda, g_{*}\right)+b_{i}+\theta_{i}\right)^{n_{i}} e^{-\left(s_{i}\left(\Lambda, g_{*}\right)+b_{i}+\theta_{i}\right)}}{n_{i} !} \\
& \times e^{-\left(\frac{1}{2} \theta^{T} V^{-1} \theta\right)}
\end{aligned}
$$

where the $s_{i}\left(\Lambda, g_{*}\right)$ is the expected number of events of the DM signal in $i$ th bin, $b_{i}$ is the respective number of background events and $n_{i}$ is the number of observed events. In our case the signal cross section for each bin is a function of the coupling $g_{*}$ and the scale $\Lambda$. For practical purposes we consider three different benchmark values for $g_{*}=4 \pi, 6$, and 1 . The systematic uncertainties of the SM backgrounds and the DM signal are treated as nuisance parameters and they are approximated by zeromean Gaussian variables $\theta_{i}$ and a covariance matrix $V$.

We define our test statistic function as

$$
T S(\Lambda) \equiv\left\{\begin{array}{ll}
-2 \ln \frac{\mathcal{L}\left(\Lambda, \hat{\theta}_{\Lambda}\right)}{\mathcal{L}(\hat{\Lambda}, \bar{\theta})} & \hat{\Lambda} \leq \Lambda \\
0 & \hat{\Lambda}>\Lambda
\end{array},\right.
$$

where $\hat{\theta}_{\Lambda}$ is a $\theta$ vector that minimizes the logarithm of Eq. (19) for a given value of $\Lambda$. On the other hand, $\hat{\Lambda}$ and $\bar{\theta}$ are the values of $\Lambda$ and of the $\theta$ vector that globally minimize the logarithm of Eq. (19). We find the upper limit on the scale of the mediator varying $\Lambda$ until $\operatorname{TS}(\Lambda)=4$. $^{4}$

In addition to the present limits we also perform a projection of the CMS reach for an integrated luminosity of $300 \mathrm{fb}^{-1}$. For this projection we assume a Gaussian likelihood, that the number of background events scales with the luminosity and that the uncertainty on the background scales as the square root of the luminosity. We set a lower background limit to be $1 \%$ of the background, based on postfit numbers with respective background error provided by ATLAS and CMS [5,70].

\section{RESULTS}

\section{A. Noncollider constraints}

In this section we present combined noncollider constraints for all operators under study. In particular, we obtain the bounds originating from DM DD searches, indirect $\mathrm{DM}$ searches from the $\mathrm{CMB}$ and relic density assuming the freeze-out mechanism and standard cosmology. The results in this section are obtained with three different tools: micrOMEGAs [49], a modified version of the code released with [46] and runDM [33-35,50].

\footnotetext{
${ }^{4}$ Here we neglect the small difference between $2 \sigma$ exclusion and $95 \%$ C.L. exclusion.
} 

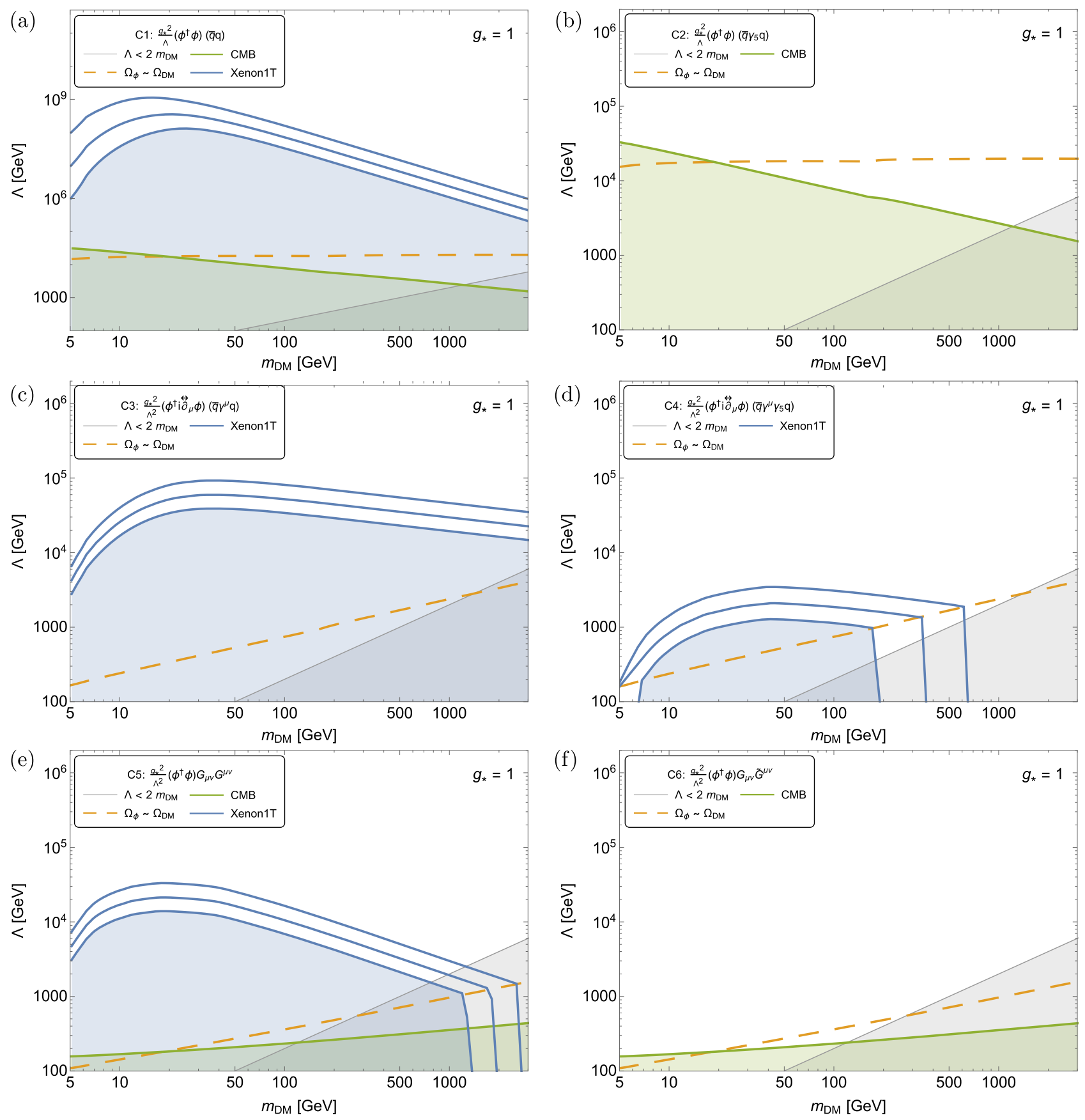

FIG. 2. Noncollider constraints on the scalar C1-C6 operators, as indicated in the panel box: (i) constraints from SI DM DD searches (excluding the shaded blue region below the lowest blue contour), (ii) constraints from relic density (excluding the region above the yellow dashed line), (iii) constraints from the CMB (excluding the shaded green area below the solid green line) and (iv) constraints from the validity of the EFT (excluding the shaded grey area, where $\Lambda<2 m_{\mathrm{DM}}$ ). The central and upper blue contour represent the central and lower values of the DM local density (see Sec. IV B).

\section{Complex scalar DM}

We start presenting in Fig. 2 the results for complex scalar DM. In all the panels, the area below the three blue curves represents the region excluded by SI DM DD experiments, taking into account the uncertainty on the local DM density as discussed in Sec. IV B. The shaded blue region is the conservative exclusion, while the middle and upper contours correspond to the center (namely the one used by the experimental collaboration) and the most optimistic exclusions, respectively. The dashed yellow line corresponds instead to the region of parameter space in which the predicted relic density matches the DM density observed by the Planck collaboration. Above the dashed yellow curve, the predicted relic density is larger than the 
experimental one. The computation is done assuming that the observed DM relic density is associated with only one particle, interacting with the SM via only one of the effective operators listed in Table I. The region excluded by the CMB measurement is indicated by a shaded green area below the solid green line. Finally, the grey-shaded region represents the region $\Lambda<2 m_{\mathrm{DM}}$, excluded by the requirement of the validity of the EFT.

From Fig. 2(a) we can learn that the $\mathrm{C} 1$ operator is strongly constrained by SI DM DD searches since this operator leads to a cross section that is neither velocity nor transferred-momentum suppressed. In fact, we can see from this panel that any scale $\Lambda$ below $1000 \mathrm{TeV}$ is excluded even for $m_{\mathrm{DM}}=1 \mathrm{TeV}$. Moreover, the other two blue contours associated with the astrophysical uncertainties show that the bounds can be tightened by almost an order of magnitude. Therefore, it is important to be conservative to make a robust exclusion of the parameter space, as we do in the present work. We can also see from Fig. 2(a) that the relic density data exclude $\Lambda$ above $20 \mathrm{TeV}$ for all DM mass range under consideration, since in this region the $\phi$ particle would over-close the universe. Notwithstanding, one should note that this bound is quite model dependent, e.g., adding an additional particle that could coannihilate with the DM could change the relic density dramatically. Finally, the CMB measurement leads to quite strong bounds that exclude $\Lambda$ below $1-10 \mathrm{TeV}$ for $g_{\star}=1$, depending on the DM mass. This is due to the fact that the $\mathrm{C} 1$ operator gives rise to s-wave annihilation cross section, which is strongly constrained by the CMB bound.

We show in Fig. 2(b) the noncollider constraints on the operator $\mathrm{C} 2$. As expected, the $\mathrm{CMB}$ and relic density bounds on $\mathrm{C} 2$ are identical to those on $\mathrm{C} 1$. The SI DM DD constraints are absent, since this operator violates parity. As mentioned in the end of Sec. III, the limits on the relevant C4, C6 momentum suppressed operators from DM direct and indirect detection searches are quite negligible (of the order of $10 \mathrm{GeV}$ on $\Lambda$ ) so we do not present them here. The limits on $\mathrm{DD}$ for the operator $\mathrm{C} 4$ originate from the running. Therefore the complementary limit from $\mathrm{CMB}$ plays a crucial role for these operators, excluding the parameter space below the solid green line for $g_{\star}=1$. The unconstrained window can be tightened for DM masses above $\simeq 20 \mathrm{GeV}$ using the relic density information if we assume that there is no process that leads to DM coannihilation.

We display in Fig. 2(c) the limits for the operator C3, that contains a vector quark current. The bounds coming from SI DM DD are weaker than those on the operator C1 since $\mathrm{C} 3$ is a dimension six operator, while $\mathrm{C} 1$ is dimension five. The uncertainty on this constraint is roughly a factor of 3 due to the higher dimensionality of the operator. Moreover, the annihilation cross section for the $\mathrm{C} 3$ operator is velocity suppressed ( $\mathrm{p}$-wave), therefore the effect on the CMB spectrum is negligible. The complementarity of the relic density and DD DM bounds completely rule out WIMP models that give rise at low energy to this operators only.

The bounds on the $\mathrm{C} 4$ operator, that contains a pseudovector quark current, are shown in Fig. 2(d). At variance with the operator C2, the SI DM DD limits are nonvanishing for operator $\mathrm{C} 4$ since the running and mixing effects play an important role; for further details see Sec. III. Notice that the sudden drop of the SI DM DD constraint around $m_{\mathrm{DM}} \simeq 200 \mathrm{GeV}$ is due to the rescaling of the DM density distribution given in Eq. (16). Similarly to the operator $\mathrm{C} 3, \mathrm{C} 4$ is also velocity suppressed, and consequently it is not constrained by the CMB.

Finally, Figs. 2(e) and 2(f) present the noncollider bounds for the C5 and C6 operators, involving the gluon field strength. As we can see, there is no SD or SI DD bound on C6 due to its parity violating nature. The bound on C5 is instead important, given the large gluon content of the proton. For DM masses below $10 \mathrm{GeV}$, the bound is comparable to the one on the $\mathrm{C} 3$ operator. For larger masses, the bound weakens because the SI cross section is suppressed by an additional factor of $m_{\mathrm{DM}}^{2}$ with respect to the C3 case [see Eq. (11)]. Still, the SI bound is always the dominant one, in the region in which the EFT is valid. The constraints of the operator C6 are instead much weaker, since it does not contribute to SI DM DD, being dominated by the CMB data and relic density.

From Fig. 2 we can notice that the slope of the CMB constraint is negative for the $\mathrm{C} 1$ and $\mathrm{C} 2$ operators, in contrast with it being positive for $\mathrm{C} 5$ and $\mathrm{C} 6$. This happens because the leading term for the annihilation cross section is independent of the DM mass for $\mathrm{C} 1$ and $\mathrm{C} 2$, while it is proportional to the DM mass squared for $\mathrm{C} 5$ and $\mathrm{C} 6$. This picture is generic - if the amplitude for DM pair annihilation is not velocity suppressed, then using dimensional analysis one finds that: (a) for dimension five operators, the annihilation cross section is constant with respect to the DM mass, leading to the slight negative slope because the "effective" photon spectrum for the CMB constraint drops with the invariant mass; (b) for dimension six operators the annihilation cross section is proportional to $M_{\mathrm{DM}}^{2}$ and one observes positive slope in $\left(M_{\mathrm{DM}}, \Lambda\right)$ plane for the $\mathrm{CMB}$ constraint.

\section{Dirac fermion DM}

In Fig. 3 we present the noncollider constraints for the operators D1-D4, that exhibit scalar or pseudoscalar interactions of fermion Dirac DM with quarks. From this figure we can see the operator D1 is strongly constrained by the SI DM DD searches while the operators D2, D3, and D4 have weaker constraints from DD searches since they give rise either to momentum suppressed spin-dependent interactions (D3 and D4) or to momentum suppressed spinindependent cross section (D2). Using XENON100 constraint on $\mathcal{O}_{11}$ we have found the respective limit on D2 operator which we present in Fig. 3(b). One can see that bound on D2 from DD can be larger than the one from 

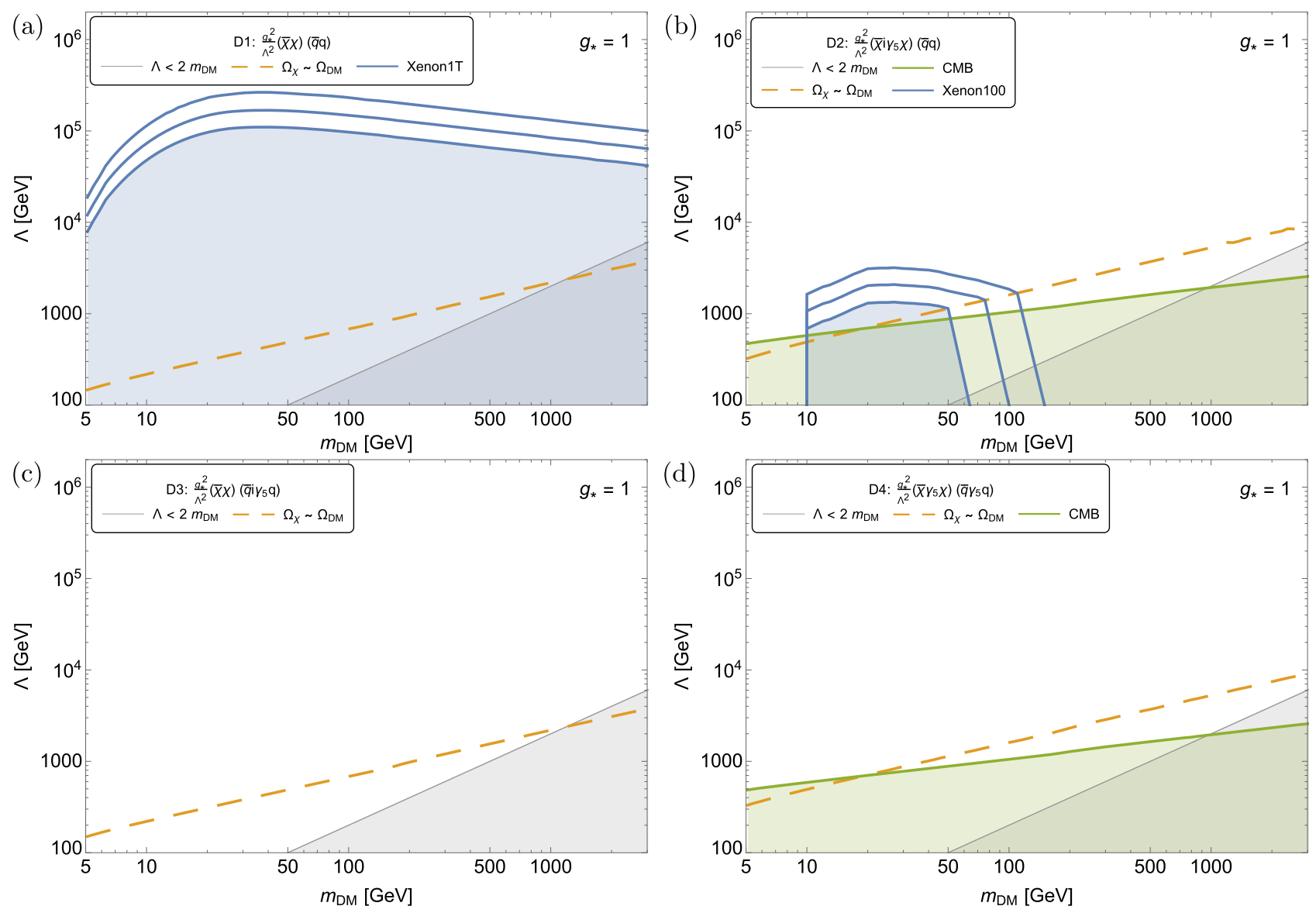

FIG. 3. As in Fig. 2, for the fermion operators involving scalar and pseudoscalar currents D1-D4.

CMB. On the other hand, bounds from DD on the operators D3 and D4 are much weaker and not presented here. Notice that the uncertainty of the SI DM DD limits on D1 is of the order of 3 since this operator is of dimension six; this is true for all operators D1-D10. The CMB data lead to constraints on the operator D2 and D4 since they exhibit an s-wave annihilation cross section, while D1 and D3 are not bound by CMB due to their $\mathrm{p}$-wave cross sections. The relic density constraint set a strong upper bound on the operators D2, D3 and D4 and rule out the operator D1 unless there is a mechanism to avoid the overproduction of DM in the early universe. As before, the theoretical consistency of our framework, i.e., the EFT validity region, becomes important just for large DM masses.

We show in Fig. 4 the noncollider limits on the fermion operators D5-D8, which contain vector and axial vector currents. The SI DM DD bound on the D5 operator is very strong, since its SI cross section is unsuppressed. On the other hand, the constraints on the operator D6 are milder, since its SI cross section is both velocity and nuclear recoil momentum suppressed. The operator D7 has in principle only SD cross sections. However, as discussed in Sec. III, the running from the higher scale mixes the operators D7 and D5, leading to SI limits on D7. The same is true for the
D8 operator, which in the running to low energy mixes into D6. Notwithstanding, the strongest limits on D8 come from the PandaX-II SD searches [62], since the D6 SI cross section is momentum suppressed in contrast with the D8 SD one. We can see from the Fig. 4 that the CMB data put stronger constraints on the D5 and D7 operators since they lead to unsuppressed s-wave annihilation cross sections. The limits on the operators D8 are much weaker since its s-wave cross section is dumped by a factor $m_{q}^{2} / m_{\mathrm{DM}}^{2}$, while the operator D6 is not limited due to its p-wave annihilation cross section.

Finally, we show in Fig. 5 the noncollider limits on the operators D9 and D10 which exhibit tensor currents. The DD cross section due to operator D9 is spin dependent, however, it is not suppressed. Notice that the conservative limit on D9 is restricted to a very narrow DM mass range. To understand this result, it is interesting to compare the operators D8 and D9, since they have the same low energy limit. Despite the numerical coefficient of D9 being twice the one of D8 [46], the constraints on D8 are stronger than the ones on D9 because the bounds on the latter are weakened by the rescaling in the relic density given in Eq. (16). On the other hand bounds on D10 operator from DD constraints are not negligible even in spite of the fact 

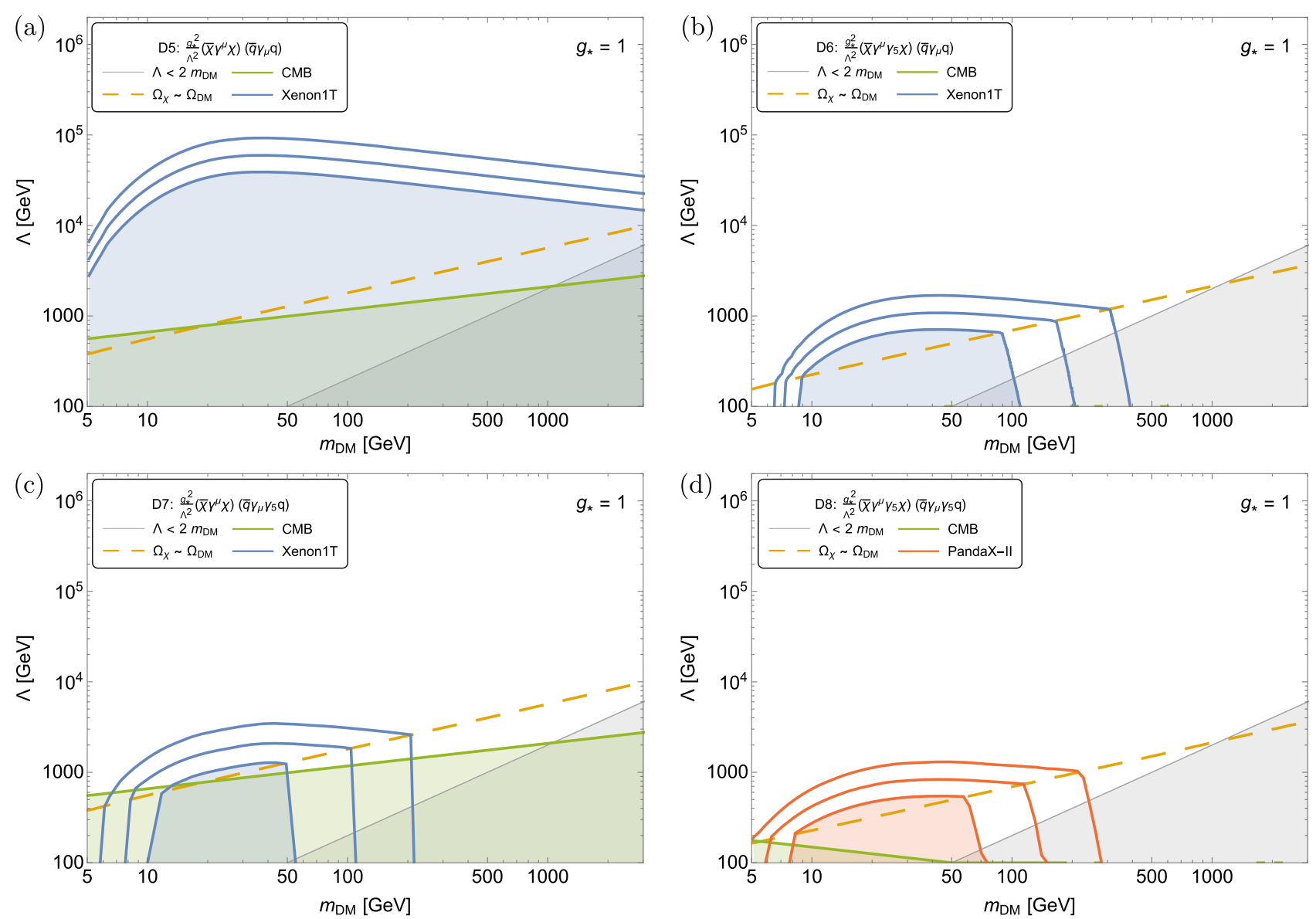

FIG. 4. As in Fig. 2 for the D5-D8 fermion operators, involving vector and vector-axial currents. The solid red lines and the respective shaded area indicate the SD DM DD bounds from PandaX-II. The uncertainty due to the different values of the local DM density is presented in an analogous way as for SI DM DD.
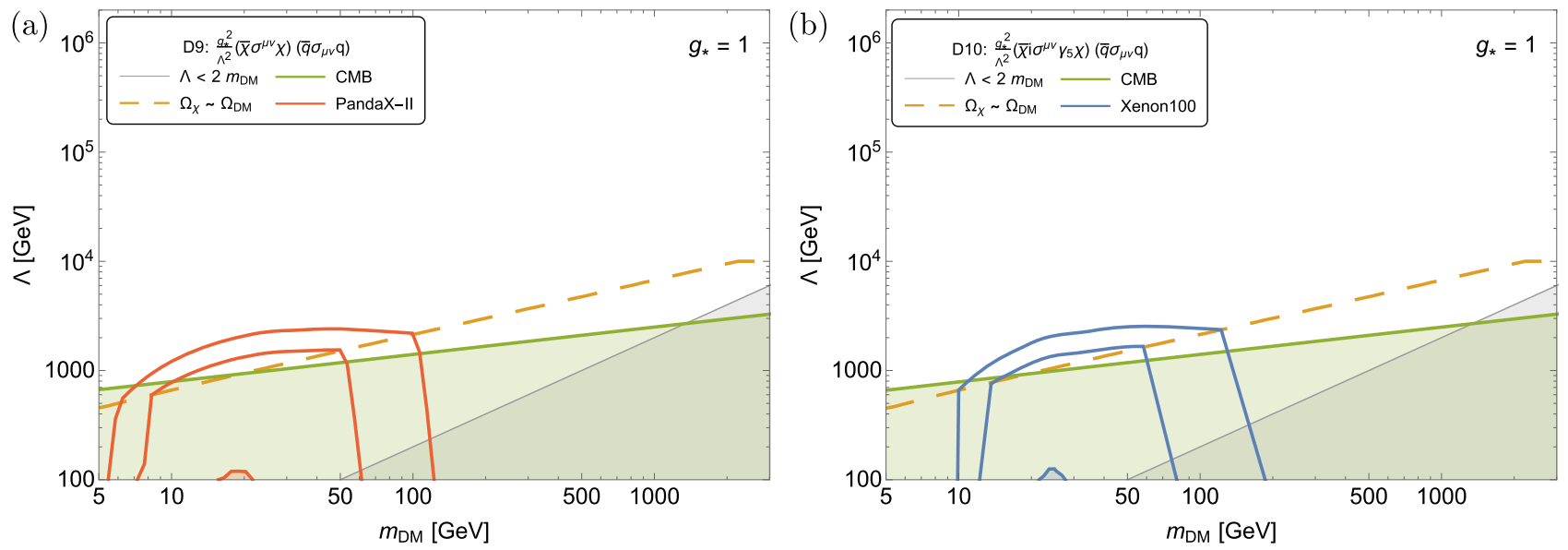

FIG. 5. As in Fig. 2 for the D9-D10 fermion operators involving tensor currents.

that DD rates are momentum suppressed. Similar to the case of D2 operator we use XENON100 constraints on $\mathcal{O}_{10}-\mathcal{O}_{12}$ nonrelativistic operators to find the limit on D10 operator presented in Fig. 5(b). One can see that this limit is weaker than the one on $\mathrm{D} 2$ but not negligible. The CMB bound on both operators D9 and D10 is important in part of the parameter space, since these operators exhibit s-wave annihilation cross sections. Moreover, for both operators, the relic density leaves only a small window below $\Lambda \sim 10 \mathrm{TeV}$ unconstrained. 
Before concluding, let us observe that also in the fermion DM case the CMB bound has positive slope, as was happening in the case of the C5 and C6 operators. Again, this is due to the fact that when the annihilation cross section is not velocity suppressed, it grows as $m_{\mathrm{DM}}^{2}$.

\section{Vector DM}

Before we present the noncollider bounds on vector DM we would like to stress that the operators V1-V12 exhibit high energy asymptotic behaviors that correspond to dimension-seven and -eight operators [22], and therefore we included additional powers of $m_{\mathrm{DM}} / \Lambda$ in their parametrization; see Table I. With this parametrization the limits on $\Lambda$ from the LHC and noncollider experiments are of the same order as the one for scalar and fermion DM operators; for further details see Ref. [22].

We present the noncollider limits on the effective operators containing vector DM in Fig. 6. When the exclusion plots are very similar, e.g., as is the case for the V2, V6, V9M, and V10M operators, we show only one representative plot. Let us discuss these results starting with DD bounds. The most stringent SI constraints are on the V1 and V3 operators, since these operators lead to nonsuppressed SI cross sections. We can see from panels 6(a) and 6(c) that for $g_{\star}=1$, the constraint on $\Lambda$ can reach $10 \mathrm{TeV}$ for large $\mathrm{DM}$ masses around $\simeq 1 \mathrm{TeV}$. Another operator for which the SI constraints are relevant is V4, although it exhibits a pseudo-vector quark current. Notwithstanding, it develops a small mixing with the V3 operator due to the running from the scale $\Lambda$ to the $1 \mathrm{GeV}$ scale. As can be seen from Fig. 6(d), the SI DD limits on V4 are weaker than those on V1 or V3; still, they dominate the constraints for DM masses in the range $\simeq 20-300 \mathrm{GeV}$. Also the V11 operator is quite constrained by SI searches, which are the dominant bound for $m_{\mathrm{DM}} \lesssim 500 \mathrm{GeV}$ (notice that, although the bounds on V11 and V12 are presented in the same panel, the SI bound applies only to the V11 operator, since V12 is parity-violating and do not contribute to the SI cross section). The other twelve operators involving vector DM either do not contribute to SI processes or their contributions are velocity/momentum suppressed, and consequently, are not bound by SI DM DD searches. It is interesting to observe that the astrophysical uncertainties amount to a factor of 2 .

The SD DM DD data can be used to constrain a few operators that lead to SD cross sections that are unsuppressed. We can see from Fig. 6(g) that for the V9P and V10P operators, the most constraining limits in the DM mass range $\simeq 20-200 \mathrm{GeV}$ stem from SD DM DD searches. On the other hand, the SD bounds on the operator V5, see Fig. 6(e), are rather weak. Nevertheless, it can be the most stringent one for DM masses between 10 and $50 \mathrm{GeV}$ depending on the local DM density.

The CMB data constrain operators exhibiting s-wave DM annihilation channels that, in the case of vector DM, are V1, V2, V5, V6, V9M, V10M, V11, and V12. In the case of the operator V1, the DM bounds are looser than the ones coming from SI DM DD, as we can see from Fig. 6(a). On the other hand, for the operator V5, the CMB limits are tighter than the conservative SD DM DD ones; see Fig. 6(e). For the remaining operators constrained by CMB data, i.e., V2, V6, V9M, V10M, and V12 the CMB bounds are the more robust ones on these operators as we can learn from Figs. 6(b) and 6(h). Nevertheless, the analyses of the relic density predicted by the operators in the last class indicate that vector operators are either ruled out $(\mathrm{V} 1, \mathrm{~V} 3$, V4, V9P, and V10P) or very strongly constrained (V2, V5, V6, V7M, V7P, V8M, V8P, V9M, V10M, V11, and V12). As remarked before, these last limits can be evaded with simple modifications of the model.

It is interesting to notice that the operators V7P, V7M, V8P, and V8M are only bound by the relic density data and EFT validity region, as we can see from Fig. 6(f). ${ }^{5}$

\section{B. LHC sensitivity and its complementarity to noncollider constraints}

In this section we present the LHC bounds on all the operators listed in Table I in the $\left(m_{\mathrm{DM}}, \Lambda\right)$ plane, analogously to what has been done for the non-collider constraints in the previous section. Applying the analysis described in Sec. IV C we obtain limits on the DM EFT operators using the CMS monojet and mono $W / Z$ (hadronically decaying) searches [5] at LHC Run 2 with an integrated luminosity of $35.9 \mathrm{fb}^{-1}$. Moreover, we also assess the LHC potential to probe the DM EFT for a projected integrated luminosity of $300 \mathrm{fb}^{-1}$.

Our results are shown in Figs. 7-11. In these figures, the red shaded area represents the LHC exclusion region at 95\% CL for $g_{\star}=1$. Furthermore, the area inside the solid orange (blue) curve is excluded at 95\% CL by the presently available CMS monojet data for $g_{\star}=6\left(g_{\star}=4 \pi\right)$. For the sake of comparison, we also display in these figures the excluded region for $g_{\star}=1$ due to noncollider searches, represented by the light purple shaded area. We do not include the relic density constraints into the "noncollider" excluded area since these bounds are model-dependent and can easily be evaded, e.g., by the addition of coannihilating DM partners. The region where the EFT approach is not valid $\left(\Lambda>2 m_{\mathrm{DM}}\right)$ is represented by the grey triangle in the right bottom side of the figures. Finally, the regions inside the dashed red, orange and blue curves represent our estimates for the future 95\% CL exclusion by the LHC with $300 \mathrm{fb}^{-1}$, taking $g_{\star}=1,6$ or $4 \pi$, respectively.

A common feature of the monojet excluded regions is that it exhibits upper and lower limits in $\Lambda$ for a fixed DM

\footnotetext{
${ }^{5}$ The relic density bounds shown correspond to the ones on V7M and V8M. The limits on V7P and V8P are very similar differing by the fact that the line describing this limit starts at $m_{\mathrm{DM}} \sim 20 \mathrm{GeV}$.
} 

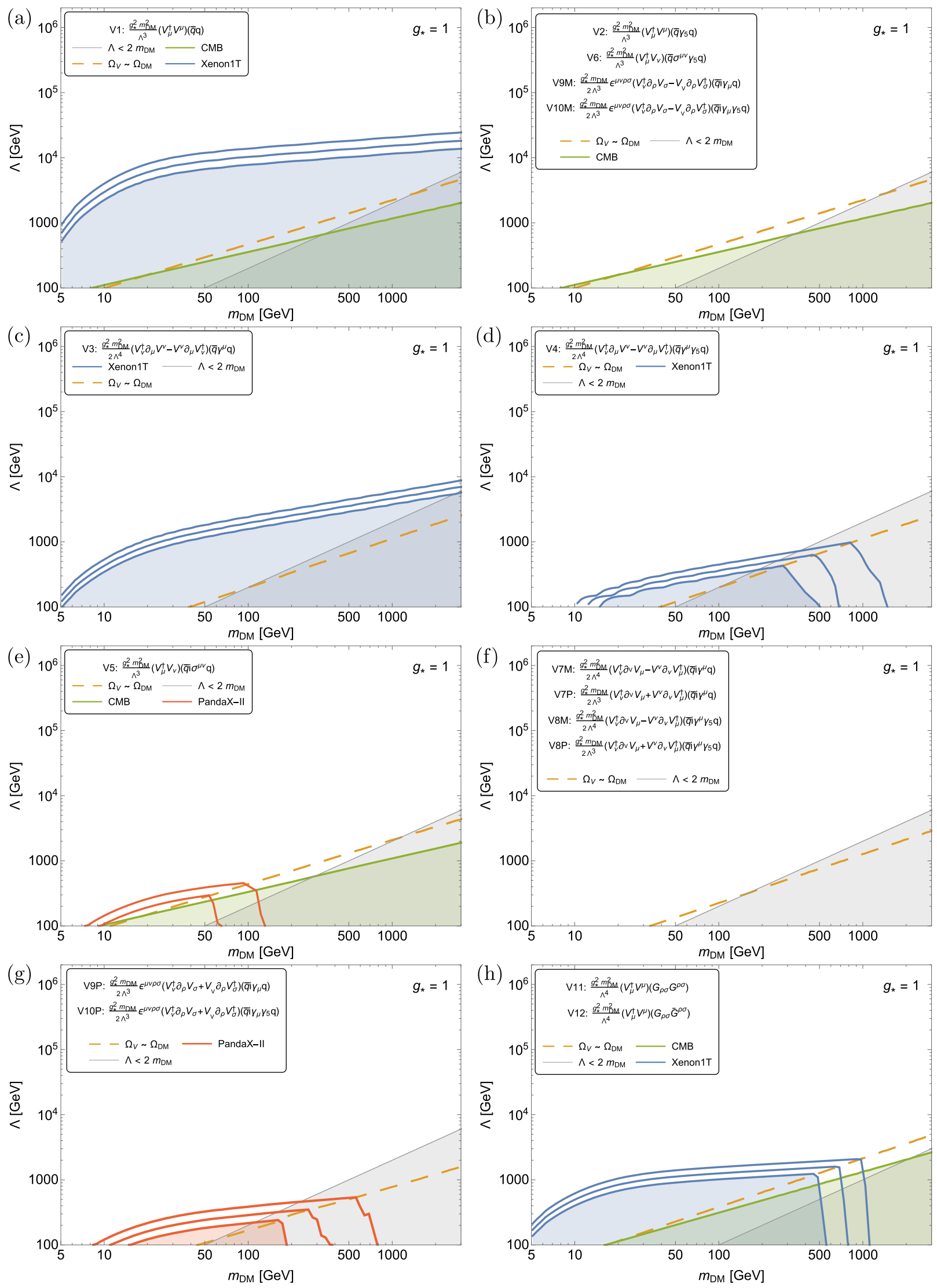

FIG. 6. Noncollider constraints (DD, CMB, relic density, and validity of the EFT) for the operators involving vector DM. We group the operators with sensibly similar bounds. The conventions are same of Fig. 4. Notice that in panel (h) the SIbound applies only to the V11 operator. 

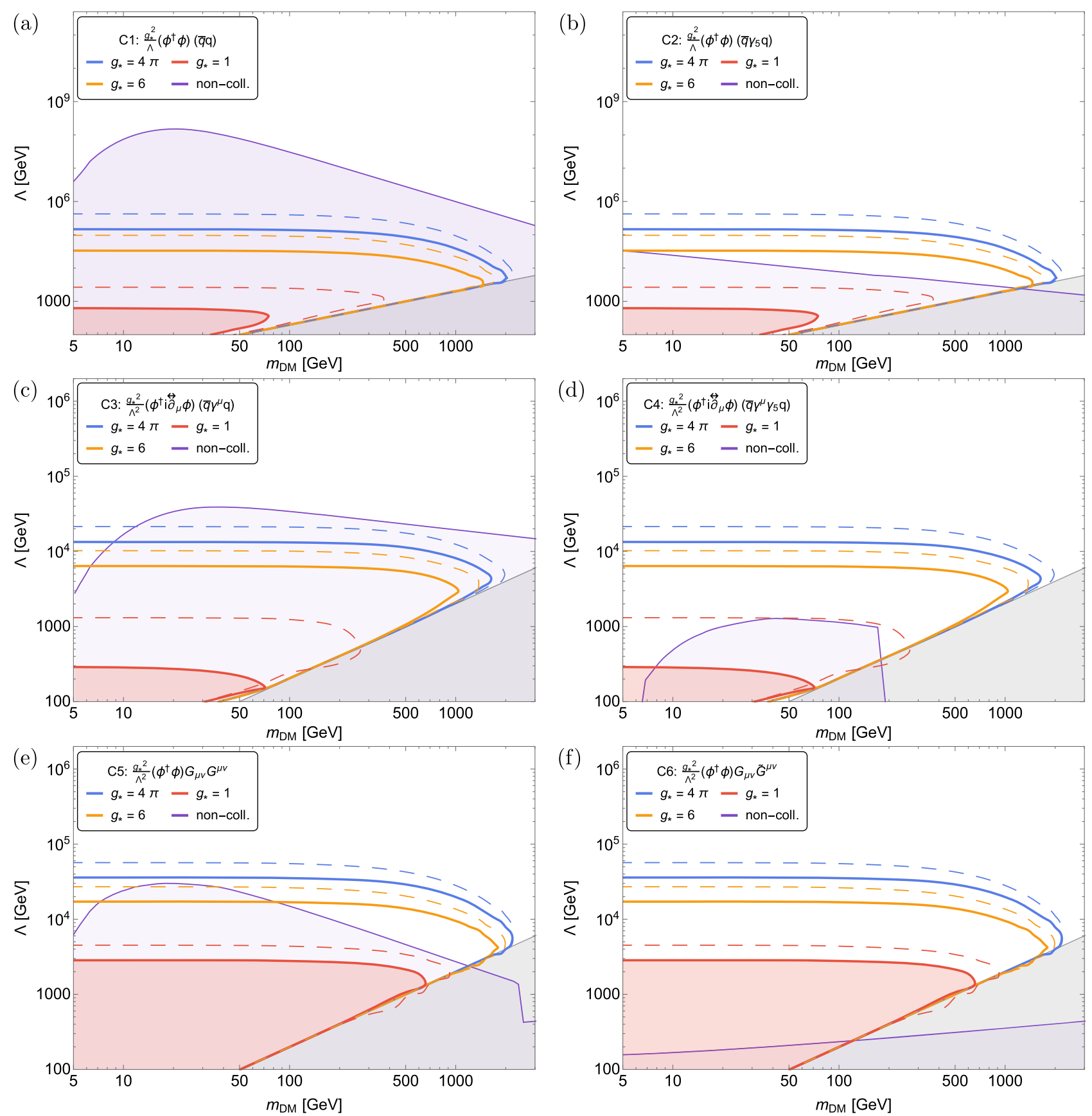

FIG. 7. LHC monojet constraints on EFT operators with scalar DM, as indicated in each panel. The area inside the red, orange, and blue solid curves is excluded by current LHC data at 95\% CL for $g_{\star}=1,6$ and $4 \pi$, respectively. The projected LHC limits for $300 \mathrm{fb}^{-1}$ are indicated by dashed thin lines. The combined exclusion regions from CMB and DM DD searches for $g_{\star}=1$ are given by the lightpurple area below the purple curve. The grey triangular region marks the region where the EFT approach is not valid.

mass. The lower limit is caused by the cut given in Eq. (18), that guarantees that the EFT is applied only within its validity limit. Furthermore, this cut has an important effect when the bound on $\Lambda$ is low. On the other hand, for higher values of $\Lambda$ (above few $\mathrm{TeV}$ ) the impact of this cut on the cross sections is negligible for scalar operators. We will see below that there are also important differences in the effects of this cut for different DM spin cases since the DM pair invariant mass distributions depend strongly on the DM spin, as discussed in Ref. [22].

\section{Complex scalar DM}

We present in Fig. 7 the CMS monojet bounds on the EFT operators C1-C6 that contain scalar DM states. From the top left panel of this figure, we can learn that the LHC 
data is able to exclude up to DM masses of $\simeq 70 \mathrm{GeV}$ for $\Lambda \lesssim 600 \mathrm{GeV}$ and $g_{\star}=1$. We can also see how these bounds evolve with the coupling $g_{\star}$ : as $g_{\star}$ varies, the upper limit on $\Lambda$ does not scale as $g_{\star}^{2}$ as naively expected. The reason for this behavior is the cut in Eq. (18), that reduces more significantly the available phase space for smaller $g_{\star}$ since this is associated to smaller $\Lambda$ upper limits. This behavior is true for all operators containing vector and pseudo-vector quark currents. Moreover, this panel shows that the conservative noncollider bounds are the strongest ones for $g_{\star}=1$ This situation persists even when a larger integrated luminosity is accumulated.

The top right panel of Fig. 7 shows the collider bounds on the operator $\mathrm{C} 2$. As we can see, the collider limits on $\mathrm{C} 1$ and $\mathrm{C} 2$ are the same. Moreover, the most stringent limits on this operators originates from noncollider data, irrespective of the integrated luminosity. In addition, from the middle left panel of this figure we see that results for the operator $\mathrm{C} 3$ are similar to the ones for $\mathrm{C} 1$ and $\mathrm{C} 2$, except that the collider limit on $\Lambda$ is reduced.

The scale of $\Lambda$ which the LHC can probe for scalar DM EFT operators strongly depends on the effective operator: it is about $0.6 \mathrm{TeV}$ for the $\mathrm{C} 1$ and $\mathrm{C} 2$ operators, it is only about $0.3 \mathrm{TeV}$ for the $\mathrm{C} 3$ and $\mathrm{C} 4$ operators, and it is about $3 \mathrm{TeV}$ for the $\mathrm{C} 5$ and $\mathrm{C} 6$ operators. The LHC searches are the least sensitive for the operators $\mathrm{C} 3$ and $\mathrm{C} 4$ for two reasons: first of all, these operators contain explicit momentum dependence through the derivative, and LHC cuts on the monojet are not hard enough to enhance this operator. Second, the invariant DM pair mass distribution is shifted to higher values than for other scalar DM operators (see detailed discussion in Ref. [22]), therefore the cut given by the Eq. (18) reduces their signal more then for other EFT operators with scalar DM.

From Fig. 7 we can also see that LHC plays an important complementary role in probing the DM parameter space for the $\mathrm{C} 4$ and $\mathrm{C} 6$ operators. Notice that the LHC searches are especially important to test the operator C6 that involve gluons. In fact, the sensitivity of noncollider experiments to the EFT parameter space for this operator is very poor, as one can see from Fig. 7(f). Moreover, even for the operators $\mathrm{C} 1, \mathrm{C} 2$, and $\mathrm{C} 3$, the LHC could help elucidate the nature of $\mathrm{DM}$ by independently probing the DM parameter space, especially if some DM signals would take place at collider and noncollider experiments.

In the case that the DM interactions are stronger, e.g., $g_{\star}=4 \pi$, we can scale the CMB and DD limits shown in Fig. 7 by $g_{\star}^{2}\left(g_{\star}\right)$ for dimension 5 (6) operators to compare with the LHC results. The conclusions for $g_{\star}=4 \pi$ are the same as the ones above for $g_{\star}=1$, except for the range of masses where the DM DD is dominant that shrinks to the interval 20-140 GeV. Moreover, we project that the LHC limits on $\mathrm{C} 4$ will be the strongest ones for an integrated luminosity of $300 \mathrm{fb}^{-1}$.

\section{Dirac fermion DM}

From Fig. 8 we can see that the LHC monojet data exclude DM masses up to $\simeq 150-200 \mathrm{GeV}$ and $\Lambda \lesssim$ $900 \mathrm{GeV}$ for the operators containing scalar and pseudoscalar quark currents and $g_{\star}=1$. Furthermore, in the case of effective operators containing vector and pseudovector quark currents, the LHC excluded region is slightly larger for $g_{\star}=1: m_{\mathrm{DM}} \lesssim 200 \mathrm{GeV}$ and $\Lambda \lesssim 1.1 \mathrm{TeV}$. In the case of couplings approaching the strongly interacting regime $\left(g_{\star}=4 \pi\right)$ the exclusion region is extended to $m_{\mathrm{DM}} \lesssim$ $2 \mathrm{TeV}$ and $\Lambda \lesssim 20 \mathrm{TeV}$ for all operators D1-D8.

Figure 8 also allows us to see the complementarity between the collider and noncollider searches. First of all, the DM SI DD bounds are more stringent than the LHC ones for operators that possess unsuppressed contributions to DM SI DD, i.e., D1 and D5; see panels (a) and (e) of this figure. On the other hand, the LHC bounds are stronger than the noncollider ones for the operators D6 and D8 since these operators only exhibit velocity suppressed contributions to DM SI DD searches. The same conclusion applies to the operator D3 that is not limited by neither the CMB data nor the DD searches. Moreover, the CMB data and the LHC monojet searches complement nicely each other for the operators D2, D4, and D7 because the LHC searches dominates the bounds for DM masses smaller than $\simeq 50 \mathrm{GeV}$ while the $\mathrm{CMB}$ limits are stronger above this mass.

Figure 9 depicts the LHC limits on the operators D9 and D10 that contain a tensor quark current. The LHC monojet searches excludes the region $m_{\mathrm{DM}} \lesssim 600 \mathrm{GeV}$ and $\Lambda \lesssim$ $3 \mathrm{TeV}$ for these operators. For larger couplings $g_{\star}=4 \pi$ the region is expanded to $m_{\mathrm{DM}} \lesssim 2 \mathrm{TeV}$ and $\Lambda \lesssim 35 \mathrm{TeV}$. For the operator D9 and $g_{\star}=1$, the CMB bounds dominates in a small region for $m_{\mathrm{DM}} \gtrsim 600 \mathrm{GeV}$ while the LHC limits are stronger below this mass. The most stringent bounds on the operator D10 have three different sources depending on the DM mass for $g_{\star}=1$. At low DM masses $\simeq 15 \mathrm{GeV}$ the bounds are dominated by the LHC searches while for heavier masses $\gtrsim 300 \mathrm{GeV}$ the most important constraints come from the CMB data. On the other hand, the SI limits are more stringent in the mass window $15 \lesssim m_{\mathrm{DM}} \lesssim 300 \mathrm{GeV}$.

As for the case of scalar DM, DD and CMB limits on the Dirac fermion DM rescale as $g_{\star}^{2}\left(g_{\star}\right)$ for dimension 5 (6) operators. In the case of $g_{\star}=4 \pi$ the region where the $\mathrm{CMB}$ constraints are more stringent than the collider ones on D2, D4, D7, and D10 changes to DM masses in excess of $350,350,400$, and $620 \mathrm{GeV}$ respectively. In the case of the other effective operators for Dirac fermion DM the conclusions remain the approximately the same for the value of $g_{\star}$.

\section{Vector DM}

We display in Figs. 10 and 11 the present constraints on vector DM operators that stem from the LHC monojet searches. Notice that the LHC cross sections do not change significantly when we replace a current by the corresponding 

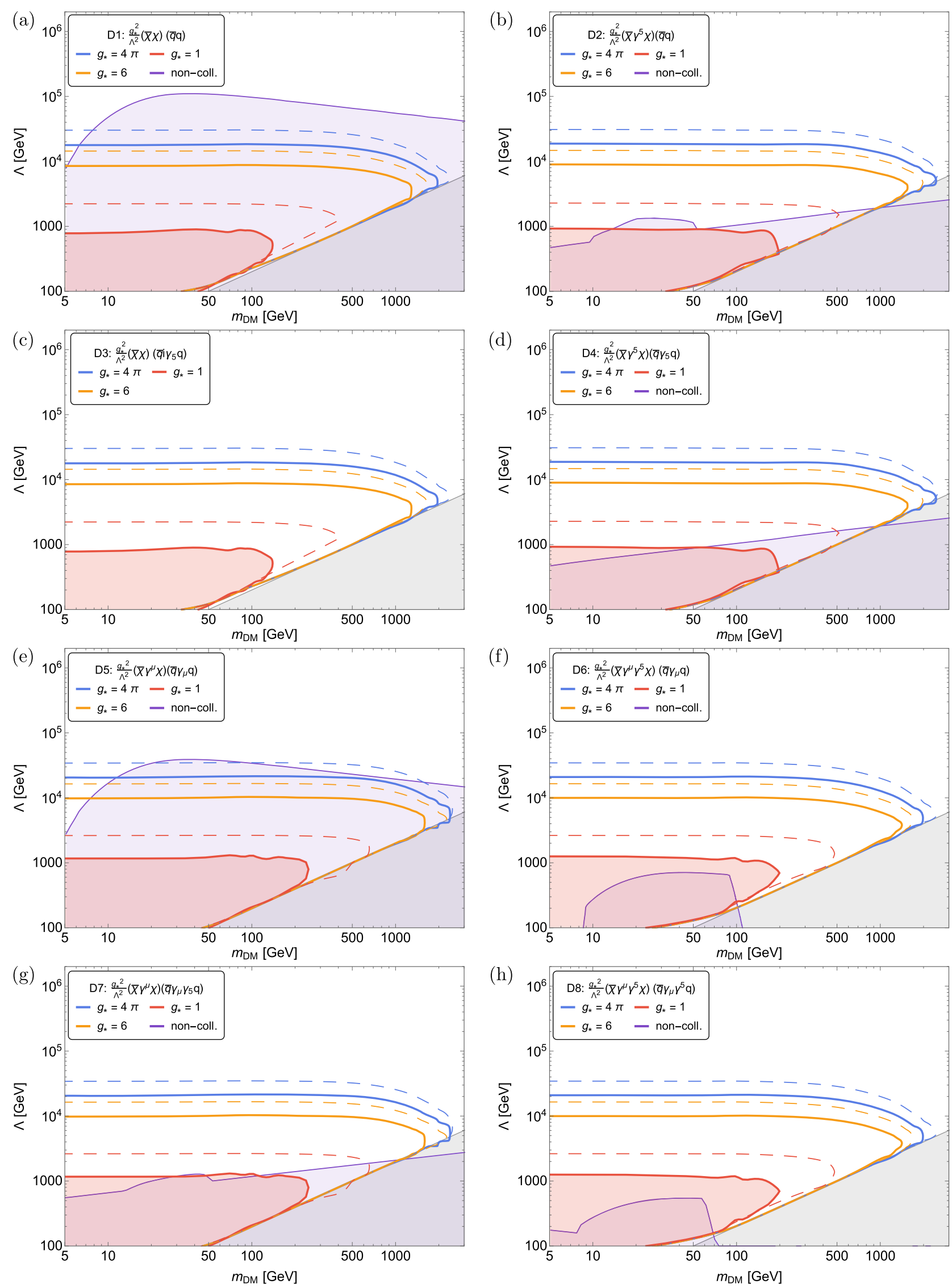

FIG. 8. Constraints from the LHC monojet searches on the operators D1-D8 that contain Dirac fermion DM. The conventions are as in Fig. 7. 

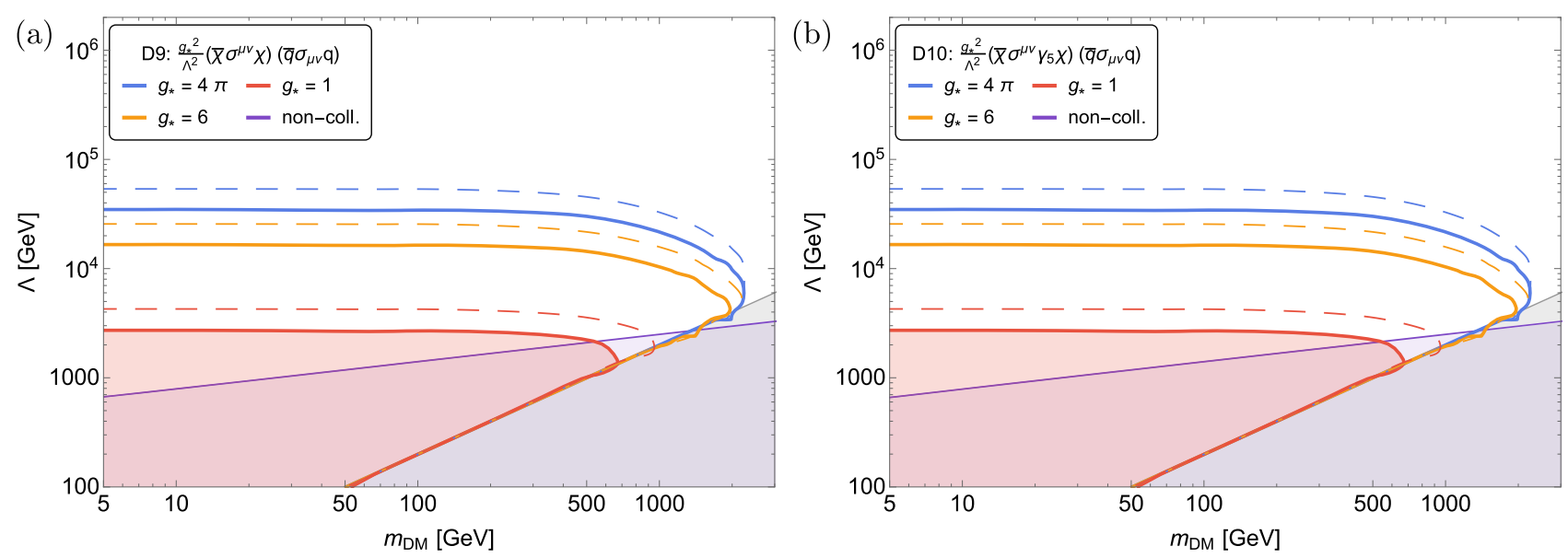

FIG. 9. Constraints from the LHC monojet searches on the operators D9 and D10 that contain Dirac fermion DM. The conventions are as in Fig. 7.
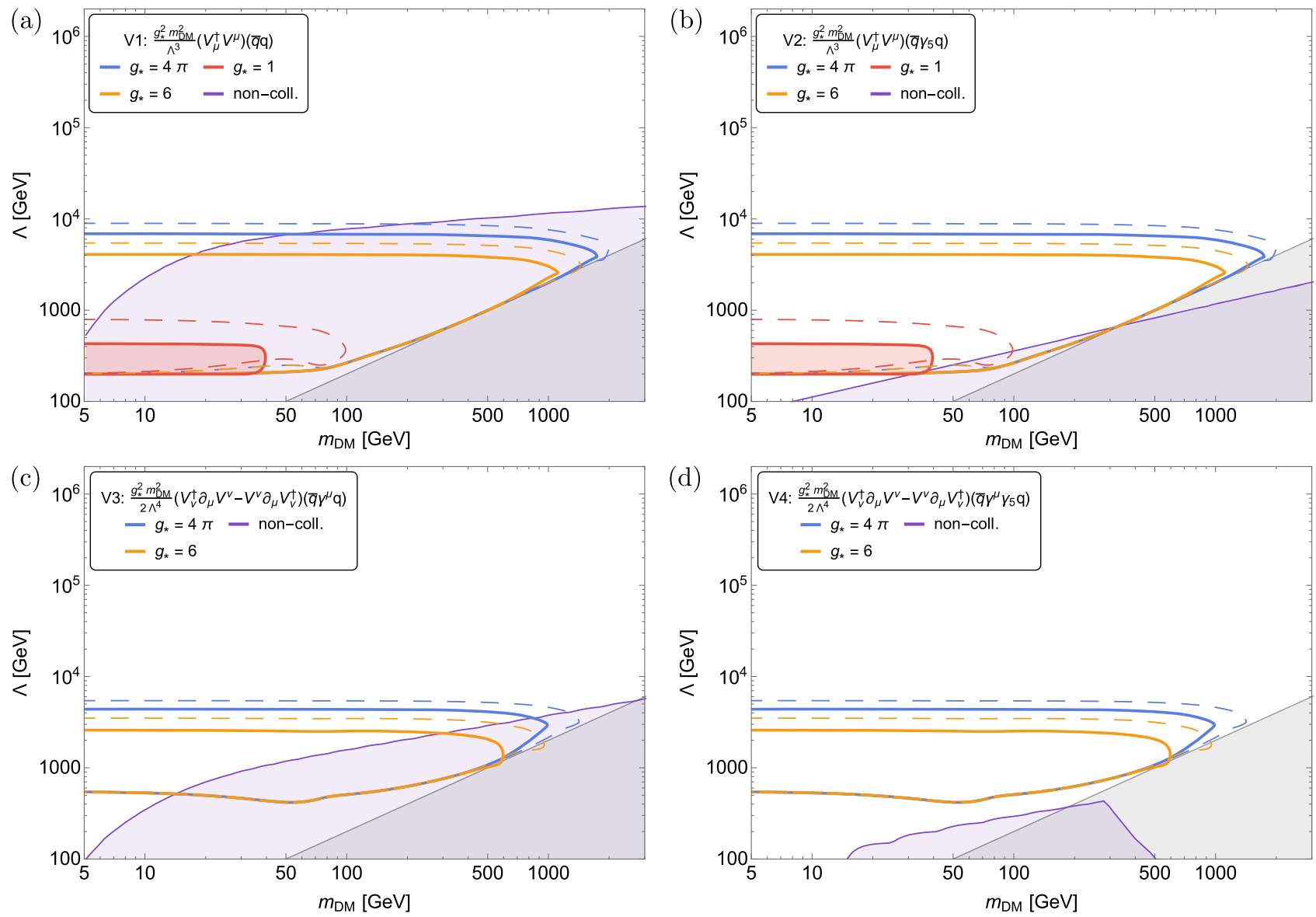

FIG. 10. Constraints from LHC monojet searches on the EFT operators V1, V2, V3, and V4 that exhibit vector DM. The conventions are as in Fig. 7.

pseudo one. Consequently, we do not present the results for the pseudocurrents unless their noncollider constraints are different.

From the top panels of Fig. 10 we learn that the LHC monojet constraints on the operators V1 and V2 are equal: for $g_{\star}=1$, the excluded region is given by $m_{\mathrm{DM}} \lesssim 40 \mathrm{GeV}$ and $100 \leq \Lambda \leq 400 \mathrm{GeV}$. As $g_{\star}$ increases to $4 \pi$, the excluded region becomes $m_{\mathrm{DM}} \lesssim 2 \mathrm{TeV}$ and $100 \leq \Lambda \leq$ $9000 \mathrm{GeV}$. Notice that the lower limit on $\Lambda$ does not depend on the coupling $g_{\star}$. Since the V1 contribution to the SI DM 

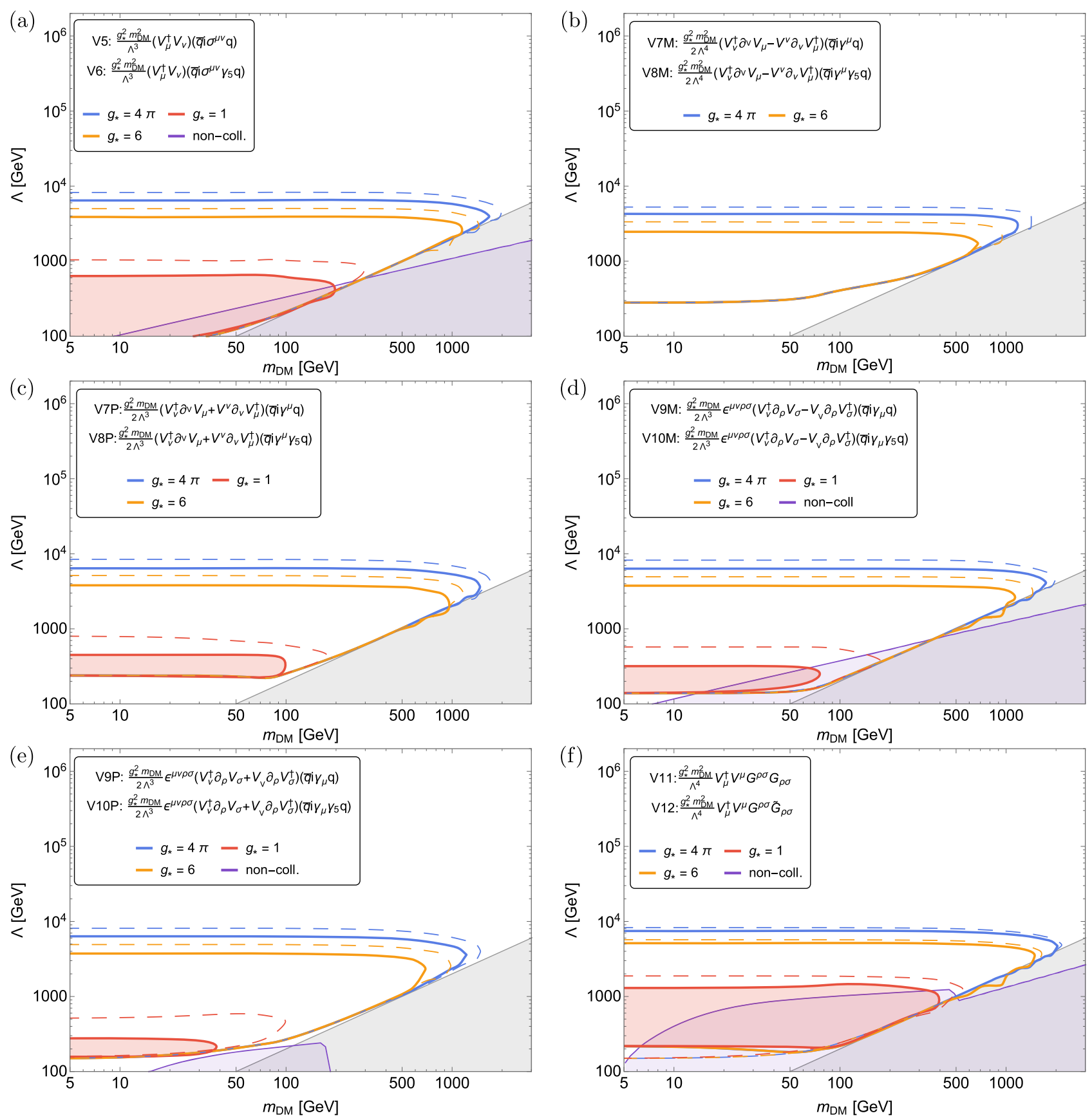

FIG. 11. Constraints from LHC monojet searches on the EFT operators V5, V6, V7M, V8M, V7P, V8P, V9M, V10M, V9P, V10P, V11, and V12 that exhibit vector DM. The conventions are as in Fig. 7.

DD is unsuppressed the strongest bound on this operator comes from the DD searches. On the other hand, for the operator V2, the collider and noncollider limits are clearly complementary: the collider limits dominate for DM masses smaller than $40 \mathrm{GeV}$ while the $\mathrm{CMB}$ data give rise to the most stringent limits at DM masses larger than $40 \mathrm{GeV}$. The operators V3 and V4 do not lead to any collider limit for $g_{\star}=1$ even for a larger integrated luminosity $\left(300 \mathrm{fb}^{-1}\right)$, therefore, the noncollider limits are the most important ones, showing again the synergy between low and high energy data. This lack of sensitivity on these operators for $g_{\star}=1$ originates from the shape of the invariant mass distribution of the DM pairs that is shifted towards high values. Consequently, the EFT validity cut in Eq. (18) discards a large fraction of the events in this case. This reduction of number of signal events is overcome only for larger values of coupling $g_{\star}=6(4 \pi)$ for which the LHC reach can be $m_{\mathrm{DM}} \lesssim 600$ (900) $\mathrm{GeV}$ and $\Lambda \lesssim 2.5$ (4) $\mathrm{TeV}$.

The top left panel of Fig. 11 displays the LHC monojet constraints on the operators V5 and V6, which are identical. 
For $g_{\star}=1$ the LHC bounds are the most stringent on these operators for DM masses up to $\simeq 180 \mathrm{GeV}$ and $\lambda \lesssim$ $600 \mathrm{GeV}$ while the $\mathrm{CMB}$ limits play an important role just in a small DM mass range around $200 \mathrm{GeV}$. On the other hand, we can see from the right top panel of this figure, that the operators V7M and V8M are not bound at all for $g_{\star}=1$. In the collider case the reason is the same behind the null results for the operators V3 and V4. These operators can only be probed by the LHC monojet searches for higher values of $g_{\star}$.

The left middle panel of Fig. 11 contains the bounds on the operators V7P and V8P that are only constrained by the LHC monojet searches that exclude the narrow region $m_{\mathrm{DM}} \lesssim 100 \mathrm{GeV}$ and $250 \lesssim \Lambda \lesssim 450 \mathrm{GeV}$ for $g_{\star}=1$. For higher values of the coupling, e.g., $g_{\star}=4 \pi$ this region is substantially expanded to $m_{\mathrm{DM}} \lesssim 1.3 \mathrm{TeV}$ and $250 \lesssim \Lambda \lesssim$ $6500 \mathrm{GeV}$. Once again the lower limit on $\Lambda$ depends on $g_{\star}$ and on the integrated luminosity. The limits on the operators V9M and V10M are equal and are shown in the right middle panel of Fig. 11. In this case we witness a nice complementarity between high and low energy data for these operators for $g_{\star}=1$. On one hand, the LHC monojet data excludes the region $m_{\mathrm{DM}} \lesssim 70 \mathrm{GeV}$ and $130 \lesssim \Lambda \lesssim 300 \mathrm{GeV}$. On the other hand, the CMB bounds are the strongest ones for $m_{\mathrm{DM}} \gtrsim 65 \mathrm{GeV}$.

We can see from the left lower panel of Fig. 11 that the limits on the operators V9P and V10P are rather loose, however, the noncollider and collider ones are complementary. The LHC monojet data exclude the area $m_{\mathrm{DM}} \lesssim$ $40 \mathrm{GeV}$ and $160 \lesssim \Lambda \lesssim 280 \mathrm{GeV}$, while the SD DM DD searches are more relevant for DM masses in the range 40$100 \mathrm{GeV}$. Moreover, the excluded area is increase for larger values of $g_{\star}$ or for larger integrated luminosities. Last, but not least, the limits on the operators V11 and V12 are presented in the lower right panel of this figure. In this case, the monojet constraints are more stringent than the direct detection (valid only for the operator V11) and CMB ones, excluding a large fraction of the parameter space for $g_{\star}=1: m_{\mathrm{DM}} \lesssim 400 \mathrm{GeV}$ and $200 \lesssim \Lambda \lesssim 1200 \mathrm{GeV}$. Notice that these are the only operators that the $\Lambda$ lower limits changes for higher couplings or integrated luminosities.

In brief, from Figs. 10 and 11 we learn that LHC is sensitive to a substantial numbers of operators containing vector DM, such as V2, V5 V6, V7P, V8P, V9-V12 which can not be probed at all or are poorly bound with noncollider searches. The LHC limits strongly depend on the type of the operator ranging from few hundred $\mathrm{GeV}$ (V1, V2, V5,V6, V7P, V8P, V9M, V9P, V10M, V10P) to about 1.3 TeV (V11,V12). Moreover, we also assess the impact of the EFT validity cut in Eq. (18) for vector DM, a fact that was not explored in previous studies.

In this scenario, the $\mathrm{DD}$ and $\mathrm{CMB}$ constraints rescale at a fixed DM mass as $g_{\star}^{2 / 3}$ and $g_{\star}^{1 / 2}$ for operators suppressed by $\Lambda^{3}$ and $\Lambda^{4}$ respectively. For $g_{\star}=4 \pi$, the dominance of the CMB bounds on the operators V2, V5/V6, V9M/V10M, and V11/V12 occurs for DM masses larger than 920, 1000, 850 , and $1500 \mathrm{GeV}$ respectively. Moreover, the collider limits on V3 are more stringent than the DD ones for $m_{\mathrm{DM}} \leq 50 \mathrm{GeV}$. For the other vector DM operators, the conclusions remain the same for the upper bounds on $\Lambda$. Nevertheless, there is a beautiful complementarity between the noncollider and LHC searches since the former exclude the region of small $\Lambda$ that the collider searches do not cover due to the validity of the EFT.

\section{CONCLUSIONS}

In this work we have presented accurate and up-to-date constraints on the complete set of dimension five and six operators connecting SM quarks and gluons with a DM candidate, which can be a complex scalar, a Dirac fermion or a complex vector.

We have performed a comprehensive analyses of the complementarity between collider and noncollider searches to probe DM parameter space, including LHC monojet data, bounds from SI and SD direct searches, relic density limits and CMB indirect constraints due to the injection of energy produced by DM annihilation in the early universe. Since the characteristic energy scale for LHC and direct searches differs by about six orders of magnitude, to correctly evaluate the experimental sensitivity to the DM EFT operators we have taken into account their running and mixing from the $\mathrm{TeV}$ scale to the $\mathrm{GeV}$ one. This effect is especially important for operators with pseudo-vector SM quark current (C4, D6, D7, and V4 in Table I) which, mixing into operators with vector SM quark current, develop a SI cross section. Another important point has been to take into account the realistic uncertainty in the DM DD searches limits due to the uncertainty on the local DM density. In some cases, the uncertainty can quantitatively shift the bounds by roughly one order of magnitude, an important result that must be taken into account to properly address the excluded parameter space. As for indirect searches, we have chosen to present only the bounds coming from $\mathrm{CMB}$ data, since they are not plagued by uncertainties on the DM distribution. These bounds are particularly important when the direct detection rates are suppressed. We have also found that the EFT validity criteria $M_{\mathrm{DM}, \mathrm{DM}}<\Lambda$ plays an important role for the case of vector DM, when the invariant mass of the DM pair $M_{\text {DM,DM }}$ is typically larger than in the case of scalar and fermion DM, and there is no collider bound for relatively small values of $\Lambda$.

Our updated bounds are summarized in Figs. 7-11 for all the operators listed in Table I. In the case of scalar DM we have found an important synergy between the different experiments: while for the operators $\mathrm{C} 1$ and $\mathrm{C} 3$ (with scalar and vector quark currents, respectively) the most stringent bounds stem from SI searches, the best limits on the C6 operator (describing DM-DM-Gluon-Gluon interactions) come from the LHC mono-jet data, since this operator is 
very weakly constrained by DD rates because it is parity odd. At the same time, the most important constraints on the $\mathrm{C} 2$ operator come from the $\mathrm{CMB}$ data, while the limits on $\mathrm{C} 4$ are dominated by the LHC searches at low $m_{\mathrm{DM}}$ and by SI bounds for large DM mass values.

The overall picture for the Dirac fermion DM is similar to the one of scalar DM: there is a synergy between the collider and noncollider searches. The strongest limits on the D1 and D5 operators are due to the SI searches, while the LHC mono-jet data put the strongest constraints on the D3, D6 and D8 operators. On the other hand, the operators D2, D4, and D9 are bounded by the LHC monojet searches at small DM masses, while at larger masses the CMB data dominate the limits. ${ }^{6}$ At the same time, the best limits on the D7 and D10 operators are defined by the interplay of the LHC searches, direct detection data and CMB constraints, depending on the DM mass.

In the case of vector DM we can also see an important complementarity between the different experiments to probe DM parameter space. The most stringent limits on the V1, V3, and V4 Wilson coefficients are set by SI searches. On the other hand, the LHC monojet data provides the strongest bounds on the V5, V6, V7P, V8P, V11, and V12 operators for any value of the DM mass. Furthermore, there is a synergy between the LHC and CMB data in probing the V2, V9M and V10M operators in different DM mass regions. One should also note the interplay between the LHC monojet searches and the SD bounds in bounding the V9P and V10P operators. We have also found that notwithstanding of combination of all data there are no limits on the operators V7M and V8M for $g_{\star}=1$. There is one more important point to stress about the interplay of different data in probing vector DM operators: the lack of the LHC sensitivity to small values of $\Lambda$ discussed above in Sec. V B 3 is complemented by the potential of CMB data to probe this region. This complementarity is manifest for many operators (V1, V2, V3, V4, V9M, V10M, V9P, V10P, V11, and V12), for which CMB data partly or completely cover the lower $\Lambda$ region which LHC is unable to probe.

${ }^{6} \mathrm{DD}$ constraints for the operator D2 could be comparable to the CMB ones.
As a general remark, in our analysis we have assumed that just one operator is nonvanishing at a time, which may or may not be the case depending on the underlying theory realized in nature. Nevertheless, our studies can be easily adapted to some specific scenario where the integration of heavy mediators can lead to more than one non-vanishing Wilson coefficient. For instance, a $t$-channel scalar mediator coupled to fermion DM would generate a $(\bar{\chi} q)(\bar{q} \chi)$ effective operator. After a Fierz transformation, this operator can be written as a combination of the fermion operators D1, D4, D5, D8, and D9 [22]. According to Fig. 8, it is clear that in this case the most stringent bounds would be set by SI searches, so that at least in first approximation the bound on $(\bar{\chi} q)(\bar{q} \chi)$ coincides with the bound on D1. Of course, the validity of such a procedure must be analyzed case by case.

\section{ACKNOWLEDGMENTS}

A. B. acknowledges partial support from the STFC Grant No. ST/L000296/1. A. B. also thanks the NExT Institute, Royal Society Leverhulme Trust Senior Research Fellowship LT140094, Royal Society International Exchange Grant No. IE150682 and Soton-FAPESP Grant No. 2016/50007-0. A. B. acknowledges partial support from the InvisiblesPlus RISE from the European Union Horizon 2020 research and innovation programme under the Marie Sklodowska-Curie grant agreement No. 690575. E. B. acknowledges support from the Fundação de Ampáro à Pesquisa (FAPESP) under Grant No. 2015/25884-4. A. P. acknowledges partial support from SOTON Diamond Jubilee Fellowship as well from Royal Society International Exchanges Grant No. IE150682. O.E. is supported in part by Conselho Nacional de Desenvolvimento Científico e Tecnológico (CNPq) Grant No. 303126/2015-9 and by FAPESP Grant No. 2012/109957. G. G. dC. is supported by FAPESP process 2016/17041-0 and thanks the University of Southampton, the CERN Theoretical Physics Department, the Humboldt University, Scuola Internazionale Superiore di Studi Avanzati (SISSA), Sapienza and University of Padova for hospitality at various stages of this work. F. I. acknowledges support from the Simons Foundation and FAPESP process 2014/11070-2.
[1] P. A. R. Ade et al. (Planck Collaboration), Planck 2015 results. XIII. Cosmological parameters, Astron. Astrophys. 594, A13 (2016).

[2] G. R. Blumenthal, S. M. Faber, J. R. Primack, and M. J. Rees, Formation of Galaxies and large scale structure with cold dark matter, Nature (London) 311, 517 (1984).
[3] J. S. Bullock, T. S. Kolatt, Y. Sigad, R. S. Somerville, A. V. Kravtsov, A. A. Klypin, J. R. Primack, and A. Dekel, Profiles of dark haloes. Evolution, scatter, and environment, Mon. Not. R. Astron. Soc. 321, 559 (2001).

[4] M. Aaboud et al. (ATLAS Collaboration), Search for dark matter and other new phenomena in events with an energetic 
jet and large missing transverse momentum using the ATLAS detector, J. High Energy Phys. 01 (2018) 126.

[5] A. M. Sirunyan et al. (CMS Collaboration), Search for new physics in final states with an energetic jet or a hadronically decaying $W$ or $Z$ boson and transverse momentum imbalance at $\sqrt{s}=13 \mathrm{TeV}$, Phys. Rev. D 97, 092005 (2018).

[6] ATLAS Collaboration, Search for dark matter in events with a hadronically decaying vector boson and missing transverse momentum in $p p$ collisions at $\sqrt{s}=13 \mathrm{TeV}$ with the ATLAS detector, Technical Report No. ATLAS-CONF2018-005, CERN, Geneva, 2018.

[7] M. Aaboud et al. (ATLAS Collaboration), Search for dark matter at $\sqrt{s}=13 \mathrm{TeV}$ in final states containing an energetic photon and large missing transverse momentum with the ATLAS detector, Eur. Phys. J. C 77, 393 (2017).

[8] G. Hinshaw et al. (WMAP Collaboration), Nine-year Wilkinson microwave anisotropy probe (WMAP) observations: Cosmological parameter results, Astrophys. J. Suppl. Ser. 208, 19 (2013).

[9] M. W. Goodman and E. Witten, Detectability of certain dark matter candidates, Phys. Rev. D 31, 3059 (1985).

[10] E. Aprile et al. (XENON Collaboration), First Dark Matter Search Results from the XENON1T Experiment, Phys. Rev. Lett. 119, 181301 (2017).

[11] D. S. Akerib et al. (LUX Collaboration), Results from a Search for Dark Matter in the Complete LUX Exposure, Phys. Rev. Lett. 118, 021303 (2017).

[12] X. Cui et al. (PandaX-II Collaboration), Dark Matter Results from 54-Ton-Day Exposure of PandaX-II Experiment, Phys. Rev. Lett. 119, 181302 (2017).

[13] T. R. Slatyer, TASI lectures on indirect detection of dark matter, in Theoretical Advanced Study Institute in Elementary Particle Physics: Anticipating the Next Discoveries in Particle Physics (TASI 2016) Boulder, CO, USA, 2016 (World Scientific, Singapore, 2017).

[14] M. Ackermann et al. (Fermi-LAT Collaboration), Searching for Dark Matter Annihilation from Milky Way Dwarf Spheroidal Galaxies with Six Years of Fermi Large Area Telescope Data, Phys. Rev. Lett. 115, 231301 (2015).

[15] B. Zitzer (VERITAS Collaboration), A search for dark matter from Dwarf Galaxies using VERITAS, Proc. Sci. ICRC2015 (2016) 1225.

[16] M. L. Ahnen et al. (Fermi-LAT and MAGIC Collaborations), Limits to dark matter annihilation cross-section from a combined analysis of MAGIC and Fermi-LAT observations of dwarf satellite galaxies, J. Cosmol. Astropart. Phys. 02 (2016) 039.

[17] H. Abdallah et al. (H.E.S.S. Collaboration), Search for Dark Matter Annihilations Towards the Inner Galactic Halo from 10 Years of Observations with H.E.S.S, Phys. Rev. Lett. 117, 111301 (2016).

[18] A. Abramowski et al. (H.E.S.S. Collaboration), Search for Photon-Linelike Signatures from Dark Matter Annihilations with H.E.S.S., Phys. Rev. Lett. 110, 041301 (2013).

[19] S. Galli, F. Iocco, G. Bertone, and A. Melchiorri, CMB constraints on dark matter models with large annihilation cross-section, Phys. Rev. D 80, 023505 (2009).

[20] S. Galli, F. Iocco, G. Bertone, and A. Melchiorri, Updated CMB constraints on dark matter annihilation cross-sections, Phys. Rev. D 84, 027302 (2011).
[21] R. Contino, A. Falkowski, F. Goertz, C. Grojean, and F. Riva, On the validity of the effective field theory approach to SM precision tests, J. High Energy Phys. 07 (2016) 144.

[22] A. Belyaev, L. Panizzi, A. Pukhov, and M. Thomas, Dark Matter characterization at the LHC in the effective field theory approach, J. High Energy Phys. 04 (2017) 110.

[23] J. Goodman, M. Ibe, A. Rajaraman, W. Shepherd, T. M. Tait, and H.-B. Yu, Constraints on dark matter from colliders, Phys. Rev. D 82, 116010 (2010).

[24] J. Kumar, D. Marfatia, and D. Yaylali, Vector dark matter at the LHC, Phys. Rev. D 92, 095027 (2015).

[25] E. Bertuzzo, C. J. C. Barros, and G. G. di Cortona, MeV dark matter: Model independent bounds, J. High Energy Phys. 09 (2017) 116.

[26] G. Busoni, A. De Simone, E. Morgante, and A. Riotto, On the validity of the effective field theory for dark matter searches at the LHC, Phys. Lett. B 728, 412 (2014).

[27] G. Busoni, A. De Simone, J. Gramling, E. Morgante, and A. Riotto, On the validity of the effective field theory for dark matter searches at the LHC, part II: Complete analysis for the $s$-channel, J. Cosmol. Astropart. Phys. 06 (2014) 060.

[28] G. Busoni, A. De Simone, T. Jacques, E. Morgante, and A. Riotto, On the validity of the effective field theory for dark matter searches at the LHC part III: Analysis for the t-channel, J. Cosmol. Astropart. Phys. 09 (2014) 022.

[29] D. Racco, A. Wulzer, and F. Zwirner, Robust collider limits on heavy-mediator dark Matter, J. High Energy Phys. 05 (2015) 009.

[30] R. J. Hill and M. P. Solon, Universal behavior in the scattering of heavy, weakly interacting dark matter on nuclear targets, Phys. Lett. B 707, 539 (2012).

[31] M. T. Frandsen, U. Haisch, F. Kahlhoefer, P. Mertsch, and K. Schmidt-Hoberg, Loop-induced dark matter direct detection signals from gamma-ray lines, J. Cosmol. Astropart. Phys. 10 (2012) 033.

[32] L. Vecchi, WIMPs and un-naturalness, arXiv:1312.5695.

[33] A. Crivellin, F. D'Eramo, and M. Procura, New Constraints on Dark Matter Effective Theories from Standard Model Loops, Phys. Rev. Lett. 112, 191304 (2014).

[34] F. D'Eramo and M. Procura, Connecting dark matter UV complete models to direct detection rates via effective field theory, J. High Energy Phys. 04 (2015) 054.

[35] F. D'Eramo, B. J. Kavanagh, and P. Panci, You can hide but you have to run: Direct detection with vector mediators, J. High Energy Phys. 08 (2016) 111.

[36] F. Bishara, J. Brod, B. Grinstein, and J. Zupan, From quarks to nucleons in dark matter direct detection, J. High Energy Phys. 11 (2017) 059.

[37] F. Iocco, M. Pato, and G. Bertone, Evidence for dark matter in the inner Milky Way, Nat. Phys. 11, 245 (2015).

[38] M. Pato, F. Iocco, and G. Bertone, Dynamical constraints on the dark matter distribution in the Milky Way, J. Cosmol. Astropart. Phys. 12 (2015) 001.

[39] J. I. Read, The local dark matter density, J. Phys. G 41, 063101 (2014).

[40] A. M. Green, Dependence of direct detection signals on the WIMP velocity distribution, J. Cosmol. Astropart. Phys. 10 (2010) 034. 
[41] A. M. Green, Astrophysical uncertainties on direct detection experiments, Mod. Phys. Lett. A 27, 1230004 (2012).

[42] N. Bozorgnia, F. Calore, M. Schaller, M. Lovell, G. Bertone, C. S. Frenk, R. A. Crain, J. F. Navarro, J. Schaye, and T. Theuns, Simulated Milky Way analogues: implications for dark matter direct searches, J. Cosmol. Astropart. Phys. 05 (2016) 024.

[43] M. R. Buckley, Using effective operators to understand CoGeNT and CDMS-Si signals, Phys. Rev. D 88, 055028 (2013).

[44] J. Brod, B. Grinstein, E. Stamou, and J. Zupan, Weak mixing below the weak scale in dark-matter direct detection, J. High Energy Phys. 02 (2018) 174.

[45] G. Belanger, F. Boudjema, A. Pukhov, and A. Semenov, Dark matter direct detection rate in a generic model with micrOMEGAs 2.2, Comput. Phys. Commun. 180, 747 (2009).

[46] M. Cirelli, E. Del Nobile, and P. Panci, Tools for model-independent bounds in direct dark matter searches, J. Cosmol. Astropart. Phys. 10 (2013) 019.

[47] M. A. Shifman, A. I. Vainshtein, and V. I. Zakharov, Remarks on Higgs boson interactions with nucleons, Phys. Lett. 78B, 443 (1978).

[48] M. Drees and M. Nojiri, Neutralino-nucleon scattering revisited, Phys. Rev. D 48, 3483 (1993).

[49] G. Belanger, F. Boudjema, A. Pukhov, and A. Semenov, micrOMEGAs $s_{3}$ : A program for calculating dark matter observables, Comput. Phys. Commun. 185, 960 (2014).

[50] B. J. Kavanagh, bradkav/rundm: Journal release. https://doi .org/10.5281/zenodo.823249, 2017.

[51] E. Aprile et al. (XENON Collaboration), Effective field theory search for high-energy nuclear recoils using the XENON100 dark matter detector, Phys. Rev. D 96, 042004 (2017).

[52] N. Anand, A. L. Fitzpatrick, and W. C. Haxton, Weakly interacting massive particle-nucleus elastic scattering response, Phys. Rev. C 89, 065501 (2014).

[53] D. P. Finkbeiner, S. Galli, T. Lin, and T. R. Slatyer, Searching for dark matter in the CMB: A compact parameterization of energy injection from new physics, Phys. Rev. D 85, 043522 (2012).

[54] S. Galli, T. R. Slatyer, M. Valdes, and F. Iocco, Systematic uncertainties in constraining dark matter annihilation from the cosmic microwave background, Phys. Rev. D 88, 063502 (2013).

[55] C. Weniger, P. D. Serpico, F. Iocco, and G. Bertone, CMB bounds on dark matter annihilation: Nucleon energy-losses after recombination, Phys. Rev. D 87, 123008 (2013).

[56] T. R. Slatyer, N. Padmanabhan, and D. P. Finkbeiner, CMB constraints on WIMP annihilation: Energy absorption during the recombination epoch, Phys. Rev. D 80, 043526 (2009).

[57] B. Henning and H. Murayama, Constraints on light dark matter from big bang nucleosynthesis, arXiv:1205.6479.

[58] M. Kawasaki, K. Kohri, T. Moroi, and Y. Takaesu, Revisiting big-bang nucleosynthesis constraints on dark-matter annihilation, Phys. Lett. B 751, 246 (2015).

[59] A. Ibarra, B. J. Kavanagh, and A. Rappelt, Bracketing the impact of astrophysical uncertainties on local dark matter searches, arXiv:1806.08714.

[60] M. Benito, N. Bernal, N. Bozorgnia, F. Calore, and F. Iocco, Particle dark matter constraints: The effect of galactic uncertainties, J. Cosmol. Astropart. Phys. 02 (2017) 007.

[61] E. Aprile et al. (XENON Collaboration), Dark Matter Search Results from a One Ton-Year Exposure of XENON1T, Phys. Rev. Lett. 121, 111302 (2018).

[62] C. Fu et al. (PandaX-II Collaboration), Spin-Dependent Weakly-Interacting-Massive-Particle-Nucleon Cross Section Limits from First Data of PandaX-II Experiment, Phys. Rev. Lett. 118, 071301 (2017).

[63] A. Alloul, N. D. Christensen, C. Degrande, C. Duhr, and B. Fuks, FeynRules 2.0-A complete toolbox for tree-level phenomenology, Comput. Phys. Commun. 185, 2250 (2014).

[64] A. Semenov, LanHEP-A package for automatic generation of Feynman rules from the Lagrangian, Updated version 3.1, arXiv:1005.1909.

[65] J. Alwall, M. Herquet, F. Maltoni, O. Mattelaer, and T. Stelzer, MadGraph 5: Going beyond, J. High Energy Phys. 06 (2011) 128.

[66] T. Sjostrand, S. Mrenna, and P.Z. Skands, PYTHIA 6.4 physics and manual, J. High Energy Phys. 05 (2006) 026.

[67] E. Conte, B. Fuks, and G. Serret, MadAnalysis 5, a userfriendly framework for collider phenomenology, Comput. Phys. Commun. 184, 222 (2013).

[68] J. de Favereau, C. Delaere, P. Demin, A. Giammanco, V. Lemaître, A. Mertens, and M. Selvaggi (DELPHES 3 Collaboration), DELPHES 3, a modular framework for fast simulation of a generic collider experiment, J. High Energy Phys. 02 (2014) 057.

[69] CMS and T. C. Collaborations, Simplified likelihood for the re-interpretation of public CMS results, Technical Reports No. CMS-NOTE-2017-001, CERN-CMS-NOTE-2017001, CERN, Geneva, 2017.

[70] M. Aaboud et al. (ATLAS Collaboration), Search for new phenomena in final states with an energetic jet and large missing transverse momentum in $p p$ collisions at $\sqrt{s}=$ 13 TeV using the ATLAS detector, Phys. Rev. D 94, 032005 (2016). 